E5-2008-45

P. E. Zhidkov*

\title{
ON THE EIGENFUNCTION EXPANSIONS
}

ASSOCIATED WITH SEMILINEAR

STURM-LIOUVILLE-TYPE PROBLEMS

Submitted to «Nonlinear Analysis: Theory, Methods and Applications»

*E-mail: zhidkov@ theor.jinr.ru 
Жидков П.Е.

О разложениях по собственным функциям, связанных

с полулинейными задачами типа Штурма-Лиувилля

Рассматриваются полулинейные обыкновенные дифференциальные уравнения, в основном автономные в форме $-u^{\prime \prime}=f(u)+\lambda u$, дополненные различными наборами стандартных граничных условий. Здесь $\lambda-$ вещественная постоянная либо спектральный параметр. Главным образом изучаются задачи в интервале $(0,1)$. В этом случае показано, что каждая из рассматриваемых задач имеет бесконечную последовательность решений либо собственных функций. Наша цель - сделать обзор недавних результатов о свойствах базисности последовательностей этих решений либо собственных функций. В ряде случаев доказано, что такая система является базисом в $L_{2}$ (в дополнение, базисом Рисса или Бари). Кроме того, кратко рассмотрена задача на полупрямой $(0, \infty)$. В этом случае спектр задачи заполняет некоторую полупрямую и возникает аналог разложений в интеграл Фурье. Доказательства основаны главным образом на теореме Бари и, кроме того, на нашем результате общего типа, обеспечивающем достаточные условия для того, чтобы последовательность функций была базисом Рисса в $L_{2}$.

Работа выполнена в Лаборатории теоретической физики им. Н. Н. Боголюбова ОИЯИ.

Препринт Объединенного института ядерных исследований. Дубна, 2008

\section{Zhidkov P.E. \\ On the Eigenfunction Expansions Associated with Semilinear \\ Sturm-Liouville-Type Problems}

We consider semilinear second-order ordinary differential equations, mainly autonomous in the form $-u^{\prime \prime}=f(u)+\lambda u$, supplied with different sets of standard boundary conditions. Here $\lambda$ is a real constant or it plays the role of a spectral parameter. Mainly, we study problems in the interval $(0,1)$. It is shown that in this case each problem we deal with has an infinite sequence of solutions or eigenfunctions. Our aim is to review recent results on basis properties of sequences of these solutions or eigenfunctions. In a number of cases, it is proved that such a system is a basis in $L_{2}$ (in addition, a Riesz or Bari basis). In addition, we briefly consider a problem in the half-line $(0, \infty)$. In this case, the spectrum of the problem fills a half-line and an analog of the expansions into the Fourier integral occurs. The proofs are mainly based on the Bari theorem and, in addition, on our general result on sufficient conditions for a sequence of functions to be a Riesz basis in $L_{2}$. JINR.

The investigation has been performed at the Bogoliubov Laboratory of Theoretical Physics, 


\section{INTRODUCTION \\ ON THE ORIGINATION OF THE PROBLEM \\ AN OBSERVATION OF RESULTS}

In several last decades, a large progress was achieved in the study of eigenvalue and boundary value problems for equations that involve semilinear secondorder elliptic partial differential operators. There were developed deep and nice methods, in particular variational and topological ones, to answer many questions for problems of this type, such as the existence, uniqueness, a number of solutions, their qualitative behavior, etc. There were discovered wide classes of such problems whose solutions behave similar in a sense to eigenfunctions of linear self-adjoint second-order elliptic eigenvalue problems. At the same time, a question remained open about basis properties of these solutions or eigenfunctions of nonlinear problems. The author of the present work believes that this question is known to specialists in the field. Below he tries to show that it naturally arises.

So, consider, for example, the following problem:

$$
-\Delta u+c(x) u=k(x)|u|^{p-1} u, \quad u=u(x), \quad x \in \Omega \subset \mathbb{R}^{N},\left.\quad u\right|_{\partial \Omega}=0,
$$

where $\Omega$ is a bounded domain with a sufficiently smooth boundary, $c(x) \geqslant 0$ and $k(x)>0$ in $\Omega$ and $c$ and $k$ are smooth functions in the closure $\bar{\Omega} ; \Delta$ is the Laplace operator in $\mathbb{R}^{\mathbb{N}}$, and $p>1$ satisfies $p<p^{\star}$ with $p^{\star}=\frac{N+2}{N-2}$ if $N \geqslant 3$, and $p^{\star}=+\infty$ if $N=1,2$. It is well known now (on this subject, see $[14,15]$ ) that this problem has a solution $u_{0}$ positive in $\Omega$ and an infinite sequence of pairwise different solutions $\left\{u_{l}\right\}_{l=1,2,3, \ldots}$ (in fact, in $[14,15]$ a more general result is presented). These solutions obey certain variational characterization. Now, we describe it briefly.

Denote by $\mathcal{M}$ the set of all subsets of $H_{0}^{1}(\Omega) \backslash\{0\}$, where $H_{0}^{1}(\Omega)$ is the usual Sobolev space, closed and symmetric with respect to 0 (the latter means that if $M \in \mathcal{M}$ and $u \in M$, then $-u \in M)$. For any $M \in \mathcal{M}$ define $\gamma(M)$ as a minimal nonnegative integer $m$ such that there exists an odd continuous mapping of $M$ into $\mathbb{R}^{m} \backslash\{0\}$. For $u \in H_{0}^{1}(\Omega)$ set

$$
J(u)=\int_{\Omega}\left\{\frac{1}{2}|\nabla u|^{2}+\frac{1}{2} c(x) u^{2}-\frac{1}{p+1} k(x)|u|^{p+1}\right\} d x
$$


and

$$
N=\left\{u \in H_{0}^{1}(\Omega): u \neq 0 \text { and } \int_{\Omega}\left(|\nabla u|^{2}+c(x) u^{2}\right) d x=\int_{\Omega} k(x)|u|^{p+1} d x\right\} .
$$

Then, one has

$$
J\left(u_{l}\right)=\inf _{\substack{M \in \mathcal{M}, M \subset N \\ \gamma(M) \geqslant l}} \max _{u \in M} J(u) .
$$

Note also that one can change this variational principle by the following one:

$$
J\left(u_{l}\right)=\inf _{\varphi} \max _{u \in \varphi\left(S^{m-1}\right), m \geqslant l} J(u),
$$

where the infimum is taken over all odd homeomorphisms $\varphi$ of the unit sphere $S^{m-1}=\left\{x \in \mathbb{R}^{m}:|x|=1\right\}$ into $N$. Note that $\gamma\left(S^{m-1}\right)=m$. In fact, this variational principle is a variant of that by P. H. Rabinowitz $[14,15]$; the latter is used in a number of articles (see, for example, $[12,13]$ ). It is important for us that the variational characterization above of solutions $u_{l}$ can be interpreted as a «nonlinear deformation» of the well-known minimax principle for eigenfunctions of linear self-adjoint eigenvalue second-order elliptic problems.

The second our remark concerns the radially symmetric case when $\Omega=\{x \in$ $\left.\mathbb{R}^{N}:|x|<1\right\}$ and $c \equiv k \equiv 1$ in $\Omega$. In this case, under the same assumptions as earlier, the problem has a positive radially symmetric solution $\bar{u}_{0}$ and an infinite sequence of solutions $\bar{u}_{l}, l=1,2,3, \ldots$, radially symmetric in $\Omega$ and such that each $l$ th solution, regarded as a function of $r=|x|$, has precisely $l$ zeros in $(0,1)$ (for the proof, see, for example, [25]). So, in the radially symmetric case, this property of solutions is again quite similar to those of eigenfunctions of linear Sturm-Liouville operators. Note in addition that the problem above is not very simple, even in the radially symmetric case, because even the uniqueness of a solution with a given nonzero number $l$ of zeros currently is unknown.

Finishing our brief consideration of results for the model problem above, we want to note that in fact there are the essentially wider classes of nonlinear elliptic second-order problems (both containing a spectral parameter or not so), solutions of which have properties similar to those of eigenfunctions of linear self-adjoint second-order elliptic problems. So, the author of the present work believes that this similarity should lead to the natural question about basis properties of systems of solutions of these nonlinear problems.

In the early 1990s, the author had the pleasure to meet with Professor S. I. Pohozaev at the Steklov Mathematical Institute in Moscow and to discuss with him related problems. Professor S. I. Pohozaev raised the question about basis properties of eigenfunctions of nonlinear problems first. The author replied that these basis properties should hold in a nonlinear sense, we do not even know in what sense, because the problems are nonlinear. And Professor Pohozaev 
answered: «I do not know». Indeed, the results we consider in what follows concern basis properties of solutions of semilinear problems in the usual, linear, sense (these solutions form usual bases). However, the author thinks that only in the case the field will be developed an answer on the question: «Do these solutions form bases and in what sense, usual linear or «nonlinear»?» could be found.

Now, we shall make a general observation of results in the field our paper is devoted to. First of all, we should note that the problem seems to be difficult, and generally speaking, the methods and approaches for its treatment are almost not created yet. Therefore, the results obtained till now concern simpler problems of the classes mentioned above. Mainly, they are obtained in the spatial dimension 1, i.e., when differential equations are ordinary and of Sturm-Liouville type.

The list of publications on this subject is not long, too. Probably, the historically first related publication was due to K. J. Brown [3]. After that, mainly A. P. Makhmudov in [8] and in his monographs [9, 10] and also, A.S. Makin and H. B. Thompson in [11], developed and generalized this result. Below we reestablish the pioneering theorem by K. J. Brown because of its larger clearness. We do not present it in its abstract operator form, but we restrict our attention to its formulation for differential equations. So, consider two equations

$$
-\left(p u^{\prime}\right)^{\prime}+q(x) u=\lambda\left(u+\sum_{i=1}^{n} c_{i}(x) u^{k_{i}}\right)
$$

and

$$
-\left(p u^{\prime}\right)^{\prime}+q(x) u+\sum_{i=1}^{n} c_{i}(x) u^{k_{i}}=\lambda u,
$$

each of which is supplied with the boundary conditions

$$
a_{1} u(0)+a_{2} u^{\prime}(0)=0=b_{1} u(1)+b_{2} u^{\prime}(1) ; \quad a_{1}^{2}+a_{2}^{2} \neq 0, b_{1}^{2}+b_{2}^{2} \neq 0 .
$$

Here $q, c_{i}:[0,1] \rightarrow \mathbb{R}$ are continuous, $k_{i}>1$ are integer and $p(x)$ is positive and continuously differentiable in $[0,1]$ (in fact, in [3] K. J. Brown considered this problem in an arbitrary finite interval $(a, b)$, but this difference is not important). The result is the following.

Theorem 1.1 (K. J. Brown [3]). Under the above assumptions there exists a sequence of eigenfunctions $\left\{u_{n}\right\}$ of each of these two problems (1.1a), (1.2) and (1.1b), (1.2) which is a basis in $L_{2}(0,1)$.

We shall prove this result in Sec. 3. Here we remark that in this theorem the eigenfunctions of the basis, by construction in the proof, approach zero in the limit $n \rightarrow \infty$ uniformly in $x$. So, this is a result on small perturbations of a linear self-adjoint problem in a sense because $k_{i}>1$ and therefore, the nonlinear terms in each equation are $o\left(u_{n}\right)$ as $n \rightarrow \infty$ uniformly in $x$. 
Note that K. J. Brown in [3] considered in addition a similar problem in the half-line $(0, \infty)$, when the spectrum $\left\{\lambda_{n}\right\}$ of the corresponding linear problem is discrete and simple and $\lambda_{n} \rightarrow+\infty$ as $n \rightarrow \infty$. His main idea in this case is the same as in theorem 1.1: eigenfunctions of the nonlinear problem that approach zero in the limit $n \rightarrow \infty$ form a basis in $L_{2}$.

Now, we observe our recent results on the subject. Everywhere, if otherwise is not stated, we assume the following about the nonlinearity in the differential equation.

(f) Let $f=f(u): \mathbb{R} \rightarrow \mathbb{R}$ be an odd continuously differentiable function and let $f(u) / u$ be a nondecreasing function of $u>0$.

First, consider the following autonomous second-order ordinary differential equation:

$$
-u^{\prime \prime}+f(u)=\lambda u, \quad u=u(x), x \in(0,1),
$$

where $\lambda \in \mathbb{R}$ is a spectral parameter. Supply equation (1.3) with the boundary conditions

$$
u(0)=u(1)=0
$$

and with the following normalization condition:

$$
\int_{0}^{1} u^{2}(x) d x=1
$$

We shall discuss below the reasons why we included the normalization condition (1.5) in the statement of the problem. In our paper [16], the following result is established.

Theorem 1.2. Under assumption $(f)$

(a) for any integer $n \geqslant 0$ there exists a pair $\left(\lambda_{n}, u_{n}\right)$ consisting of an eigenvalue $\lambda_{n}$ and the corresponding eigenfunction $u_{n}$ of problem (1.3)-(1.5) such that $u_{n}$ possesses precisely $n$ zeros in $(0,1)$;

(b) for any integer $n \geqslant 0$ the eigenfunction $u_{n}$ that possesses precisely $n$ zeros in $(0,1)$ is unique up to the coefficient \pm 1 ;

(c) the sequence of all eigenfunctions $\left\{u_{n}\right\}_{n=0,1,2, \ldots}$ is a Bari basis in $L_{2}(0,1)$.

By definition, a Bari basis in $L_{2}(0,1)$ is a basis in this space and there exists an orthonormal basis $\left\{e_{n}\right\}_{n=0,1,2, \ldots}$ in $L_{2}(0,1)$ such that

$$
\sum_{n=0}^{\infty}\left\|u_{n}-e_{n}\right\|_{L_{2}(0,1)}^{2}<\infty .
$$

Note that in view of condition (1.5) the eigenfunctions $u_{n}$ do not go to 0 uniformly in $x$ as $n \rightarrow \infty$. Here, it is important for us that a Bari basis is in particular a basis. 
In view of this theorem, our problem (1.3)-(1.5) is «good» (or «well posed») in a sense. Note also that if one changes the normalization condition (1.5) by

$$
\int_{0}^{1} u^{2}(x) d x=A,
$$

where $A>0$ is arbitrary, then our result still holds with the only change of the term «Bari basis» by «Riesz basis» in the claim (c). Therefore, if one considers problem (1.3)-(1.4) (i.e., the above problem without the normalization condition (1.5)), then by this argument the set of all eigenfunctions becomes too wide (in this case this set will contain «many» bases). At least in this sense, it seems to be quite appropriate to include a normalization condition as (1.5) in the statement of the problem.

In the present paper, we shall not prove theorem 1.2; we only make some comments to it. Claims (a) and (b) of this result are proved in [16] and later reestablished in monograph [25]. As for the principal claim (c), we have to note that its proof in [16] contains the essential errors which fortunately can be corrected; these corrections are published in [18]. In addition, another proof of theorem 1.2(c) without these errors is established in [17]. A complete proof of theorem 1.2 is contained in [25] and therefore, we shall not establish it here.

Results similar to theorem 1.2 occur if we supply equations (1.3) and (1.4) with the normalization condition $u^{\prime}(0)=1$ : for any integer $n \geqslant 0$ this new problem has a unique eigenfunction $u_{n}$ with precisely $n$ zeros in $(0,1)$ and the sequence $\left\{u_{n} /\left\|u_{n}\right\|_{L_{2}(0,1)}\right\}_{n=0,1,2, \ldots}$ is a Bari basis in $L_{2}(0,1)$. Quite similar results still take place for equation (1.3) taken with the boundary conditions

$$
u^{\prime}(0)=u(1)=0
$$

or

$$
u^{\prime}(0)=u^{\prime}(1)=0
$$

and with the normalization condition $u(1)=1$ (for the proofs of all these results, see $[19,20])$. Methods of the analysis of these three problems are quite similar to each other, and we shall illustrate them in Sec. 3 with an example. We also mention paper [23] where an integrodifferential equation is studied.

In Sec. 4, we shall consider two problems. The first one is a problem without a spectral parameter:

$$
u^{\prime \prime}+f(u)=0, \quad u=u(x), x \in(0,1), u(0)=u(1)=0 .
$$

In this case, we assume in addition to hypothesis (f) that $f(u) / u \rightarrow+\infty$ as $u \rightarrow+\infty$ and that $f^{\prime}(0) \leqslant 0$. Again, for each integer $n \geqslant 0$ this problem has a solution $u_{n}$ unique up to the coefficient \pm 1 that possesses precisely $n$ zeros 
in $(0,1)$. One of the difficulties related to the analysis of this problem is that, unlike it takes place in the case of each previous problem, it seems to be difficult (or even impossible) to find a linear self-adjoint problem whose eigenfunctions $e_{n}$ satisfy (1.6). However, the following result takes place.

Theorem 1.3. Let the above assumptions be valid. Then, for any integer $n \geqslant 0$ problem (1.7) has a solution $u_{n}$ with precisely $n$ zeros in $(0,1)$. For each integer $n \geqslant 0$ such a solution with precisely $n$ zeros in $(0,1)$ is unique up to the coefficient \pm 1 . The sequence $\left\{u_{n}\right\}_{n=0,1,2, \ldots}$ of all solutions is a basis and the sequence $\left\{u_{n} /\left\|u_{n}\right\|_{L_{2}(0,1)}\right\}_{n=0,1,2, \ldots}$ is a Riesz basis in $L_{2}(0,1)$.

This result is proved in [21]. We shall consider this proof in Sec. 4.

Second, in Sec. 4, we shall consider briefly our result for equation (1.3) on the half-line $x>0$. We supply our equation with the boundary conditions

$$
u^{\prime}(0)=0, u(0)=p>0, \sup _{x>0}|u(x)|<\infty,
$$

where $p$ is a fixed parameter. In this case, the «spectrum» of the problem fills a half-line (note that we even have no common in use definition of the spectrum for this problem). We establish a result on a possibility of a unique expansion of an «arbitrary function» into an integral over the eigenfunctions of this problem analogous to the Fourier transform. Readers may find our proof of this result in [24]. This proof is sufficiently complicated and therefore, we do not establish it in detail in the present paper. We only outline its main idea.

A large part of proofs of the results in the present work is based on a classical result of N. K. Bari, the so-called Bari theorem. In addition, we shall apply our result giving sufficient conditions for a sequence of functions to be a Riesz basis in $L_{2}$. The proof of the latter is also based on ideas from the proof of the Bari theorem.

Finishing our introduction, we want to make some remarks. First, of course, equations (1.3) and (1.7) can be solved by quadratures. However, it seems to be not clear so far how one can analyze basis properties of systems of their eigenfunctions using such representations by quadratures for these eigenfunctions. Second, we want to discuss the normalization condition as (1.5). As the reader could already see, roughly speaking, the author has the following point of view: if a nonlinearity obeys assumption (f) and if its sign in the differential equation is such that the problem without a spectral parameter has an infinite sequence of solutions as it takes place in the case of problem (1.7), then one does not need to impose a normalization condition and he should look for basis properties of the family of solutions of the problem without a spectral parameter. While when the sign at the nonlinearity in the differential equation is opposite as in (1.3), a normalization condition should be imposed.

Observe the following. In the case of a linear self-adjoint Sturm-Liouville problem usually one does not include a normalization condition in the statement 
of the problem because if $w$ is an eigenfunction of this linear problem, then for any $\alpha \neq 0 \alpha w$ is still its eigenfunction. On the other hand, in view of this reason, one may include a normalization condition, such as (1.5) or, say, $u^{\prime}(0)=1$, in the statement of his linear self-adjoint problem explicitly. Another situation occurs in the nonlinear case. Above we tried to show that it seems to be natural to consider sometimes a nonlinear problem with a normalization condition to make this problem «good» in a sense. It is another question what normalization condition should be imposed. The author believes that this question may be solved only in the future if and when the field will become more developed. This choice may depend, for example, on the nature of a physical phenomenon leading to the corresponding mathematical problem. In this connection, the author wants to note the following. In the publications by A.P. Makhmudov [8-10] and by A.S. Makin and H. B. Thompson [11], problems in the essentially more general form than (1.3)-(1.5) or (1.7) are considered, in particular, with boundary conditions (1.2) and with nonlinearities of a more general kind than ours. A typical result in these papers is the existence of a (Riesz or Bari) eigenfunction basis for $L_{2}$. However, the author of the present work believes that, in view of this generality and because these authors do not impose a normalization condition, they do not prove a uniqueness of their bases; in fact, for their problems, there exist «a lot of» (in fact, continuum) eigenfunction bases.

As for possible applications of results on basis properties of solutions of nonlinear problems, the author thinks that if the field will continue to develop, then in the future these results may find applications. The author thinks that the results of this type can be applied in particular in the Fourier and Galerkin methods for solving differential equations.

For the convenience of readers, the author tried to include in the paper all the information from the general theory of non-orthogonal expansions in a Hilbert space necessary to read it independently of other literature. In particular, in the following we shall establish the Bari theorem. We hold a little-known and nice approach presented by Professor N. K. Bari in her pioneering papers [1,2]. We shall establish a simple and short proof of this result. In fact, this proof is a variant of that one in [2]. With this, we shorten and simplify the proof of this result established in [25], and we remove a gap in the proof occurred in [25].

The author uses this occasion to thank his colleagues for useful discussions on the subject the work is devoted to. He wants to thank especially Professor S. I. Pohozaev who contributed much for enlarging the author's interest in the field.

\section{AUXILIARY RESULTS}

First, we introduce some very simple notation. Let $L_{2}(a, b)$, where $0 \leqslant a<b \leqslant+\infty$, be the standard Lebesgue space of real-valued and square 
integrable functions $g, h, \ldots:(a, b) \rightarrow \mathbb{R}$; this space is equipped with the scalar product $(g, h)_{L_{2}(a, b)}=\int_{a}^{b} g(x) h(x) d x$ and the corresponding norm $\|g\|_{L_{2}(a, b)}=$ $(g, g)_{L_{2}(a, b)}^{1 / 2}$. Denote $L_{2}=L_{2}(0,1),(\cdot, \cdot)=(\cdot, \cdot)_{L_{2}(0,1)}$ and $|\cdot|_{2}=\|\cdot\|_{L_{2}(0,1)}$. We also introduce the space $l_{2}=\left\{a=\left(a_{0}, a_{1}, \ldots, a_{n}, \ldots\right):\|a\|_{l_{2}}<\infty\right\}$, where $a_{n} \in \mathbb{R}$ and $\|a\|_{l_{2}}=\left\{\sum_{n=0}^{\infty} a_{n}^{2}\right\}^{1 / 2}$. In addition, we equip this space with the scalar product $(a, b)_{l_{2}}=\sum_{n=0}^{\infty} a_{n} b_{n}$ making it a Hilbert space.

2.1. Basic Definitions. A Counterexample. The Bari Theorem. Now, we recall some basic definitions, partly known, we need in the following. Let $H$ be a separable Hilbert space over the field of real numbers in which, the scalar product and the corresponding norm are denoted, respectively, $(\cdot, \cdot)_{H}$ and $\|\cdot\|_{H}=(\cdot, \cdot)_{H}^{1 / 2}$. Let $\left\{h_{n}\right\}_{n=0,1,2, \ldots}$ be a sequence of elements of $H$. Then, we call this sequence a Schauder basis in $H$ (or simply a basis in $H$ ) if for any $h \in H$ there exists a unique sequence $\left\{a_{n}\right\}$ of real numbers $a_{n}$ such that $h=\sum_{n=0}^{\infty} a_{n} h_{n}$ in $H$. We call the sequence $\left\{h_{n}\right\}$ linearly independent in $H$ if the equality $\sum_{n=0}^{\infty} a_{n} h_{n}=0$ with real coefficients $a_{n}$ holds in $H$ when and only when $0=a_{0}=a_{1}=\ldots=a_{n}=\ldots$. The system $\left\{h_{n}\right\}$ is said to be complete in $H$ if for any $h \in H$ and $\epsilon>0$ there exist real coefficients $a_{n}, n=0,1, \ldots, N$, so that $\left\|h-a_{0} h_{0}+\ldots+a_{N} h_{N}\right\|_{H}<\epsilon$. We call a system which is not complete an incomplete system. A basis $\left\{h_{n}\right\}_{n=0,1,2, \ldots}$ in $H$ is called a Riesz basis in $H$ if the series $\sum_{n=0}^{\infty} a_{n} h_{n}$ with real coefficients $a_{n}$ converges in $H$ if and only if $a=\left(a_{0}, a_{1}, \ldots, a_{n}, \ldots\right) \in l_{2}$. Two sequences $\left\{h_{n}\right\} \subset H$ and $\left\{g_{n}\right\} \subset H$ are called quadratically close to each other in $H$ (or the system $\left\{h_{n}\right\}$ is called quadratically close to $\left\{g_{n}\right\}$ in $H$ ) if $\sum_{n}\left\|g_{n}-h_{n}\right\|_{H}^{2}<\infty$. A basis in $H$ is called a Bari basis in $H$ if it is quadratically close to an orthonormal basis in $H$. Note that, as it follows from the Bari theorem (see below), a Bari basis in $H$ is a Riesz basis in $H$.

Remark 2.1. Sometimes a sequence linearly independent in $H$ is called an $\omega$-linearly independent system (in particular, this terminology is used in monograph [6]). In the following, we shall use the terminology we just introduced for brevity. Defining the notion of a Riesz basis, we followed the pioneering articles 
by N. K. Bari [1,2]. In [6], another, equivalent definition is introduced. We also mention the wonderful fact that the estimates

$$
c\|a\|_{l_{2}}^{2} \leqslant\left\|\sum_{n} a_{n} h_{n}\right\|_{H}^{2} \leqslant C\|a\|_{l_{2}}^{2}
$$

with positive constants $0<c<C$ independent of coefficients $a \in l_{2}$ that sometimes are used to define Riesz bases follow from our definition of these objects; we shall see this in what follows.

Now, we want to note the following. Sometimes, a mistake occurs that the completeness and linear independence of a system $\left\{h_{n}\right\} \subset H$ together imply that this system is a basis in $H$. The following counterexample shows that, generally speaking, this is not so.

Example 2.2. Let $\left\{e_{n}\right\}_{n=0,1,2, \ldots} \subset H$ be an orthonormal basis in $H$, and consider the sequence $h_{0}=e_{0}, h_{n}=e_{n}+e_{0}$ for $n=1,2,3, \ldots$ It is easily seen that the system $\left\{h_{n}\right\}_{n=0,1,2, \ldots}$ is complete and linearly independent in $H$. Let us prove that this system is not a basis in $H$. Take an $h=\sum_{n=0}^{\infty} c_{n} e_{n} \in H$, where $c=\left(c_{0}, c_{1}, \ldots, c_{n}, \ldots\right) \in l_{2}$ is such that the series $\sum_{n=0}^{\infty} c_{n}$ is nonconvergent, and suppose that $h=\sum_{n=0}^{\infty} a_{n} h_{n}$ in $H$. Then, since the basis $\left\{e_{n}\right\}$ is orthonormal, we find step by step multiplying the last relation by $e_{n}, n=1,2,3, \ldots$, in $H$ : $a_{1}=c_{1}, a_{2}=c_{2}, \ldots, a_{n}=c_{n}, \ldots$ and hence, it must be $a_{0}=c_{0}-\sum_{n=1}^{\infty} c_{n}$, which is a contradiction. Therefore, $\left\{h_{n}\right\}_{n=0,1,2, \ldots}$ is not a basis in $H$.

One of the tools we use in the present paper is the Bari theorem. It was established for the first time by Professor N. K. Bari in [1,2] and later reestablished in a number of publications (see, for example, $[6,25]$ ).

Theorem 2.3 (Bari theorem). Let $H$ be a separable Hilbert space over the field of real numbers, the scalar product and norm in which are denoted, respectively, $(\cdot, \cdot)_{H}$ and $\|\cdot\|_{H}=(\cdot, \cdot)_{H}^{1 / 2}$. A sequence of elements of this space linearly independent and quadratically close to a Riesz basis in this space is a Riesz basis in $H$.

Proof. Here, we follow the pioneering article by N.K. Bari [2]. Let a sequence $\left\{h_{n}\right\}_{n=0,1,2, \ldots}$ be linearly independent and quadratically close to a Riesz basis $\left\{e_{n}\right\}_{n=0,1,2, \ldots}$ in $H$. Then, for $a=\left(a_{0}, a_{1}, \ldots, a_{n}, \ldots\right) \in l_{2}$, we have

$$
\left\|\sum_{n=m+1}^{m+p} a_{n}\left(e_{n}-h_{n}\right)\right\|_{H}^{2} \leqslant \sum_{n=m+1}^{m+p} a_{n}^{2} \sum_{n=m+1}^{m+p}\left\|e_{n}-h_{n}\right\|_{H}^{2} \rightarrow 0
$$


as $m \rightarrow \infty$ uniformly with respect to $p \geqslant 0$. Hence, the series $\sum_{n=0}^{\infty} a_{n}\left(e_{n}-h_{n}\right)$ converges in $H$.

We associate the element $h=\sum_{n=0}^{\infty} a_{n} e_{n} \in H$ to each $a=\left(a_{0}, a_{1}, \ldots, a_{n}, \ldots\right) \in$ $l_{2}$. Clearly, the map $a \rightarrow h$ is a linear one-to-one correspondence of $l_{2}$ onto $H$. In addition, it follows from Banach's general theory of bases in the Banach spaces that the coefficients $a_{n}$ which are obviously linear functionals depending on $h \in H$ are in addition continuous functionals (a simple and short proof of this claim is presented, for example, in [7]). Below we do not prove this fact referring the reader to other literature, for example, to book [7], for its proof.

For $h \in H$, denote $A_{N}: H \rightarrow l_{2}$ so that $A_{N} h=a^{N}=\left(a_{0}, a_{1}, \ldots, a_{N}, 0,0, \ldots\right) \in$ $l_{2}$. Then, $A_{N}$ are linear continuous operators from $H$ into $l_{2}$. Since for each $h \in H$ one has $\sum_{n} a_{n}^{2}<\infty$, for each $h \in H$ the sequence $\left\{A_{N} h\right\}_{N=1,2,3, \ldots}$ is bounded in $l_{2}$, therefore, by the Banach-Steinhaus theorem, the sequence of bounded linear operators $\left\{A_{N}\right\}_{N=1,2,3, \ldots}$ from $H$ into $l_{2}$ is bounded uniformly in $N$. Thus, the linear operator $A$ that maps $h \in H$ into $a=\left(a_{0}, a_{1}, \ldots, a_{n}, \ldots\right) \in l_{2}$ is bounded. So, there exists $M>0$ such that $\|A h\|_{l_{2}} \leqslant M\|h\|_{H}$ for any $h \in H$.

Let $F=\sum_{n=0}^{\infty} a_{n}\left(e_{n}-h_{n}\right)$, and denote $F=U h$, where $h=\sum_{n} a_{n} e_{n}$. Then, the operator $U: H \rightarrow H$ is linear and it is well-defined onto the whole space $H$. Let us prove that this operator $U$ is completely continuous in $H$. Denote $B_{R}=B_{R}(0)=\left\{g \in H:\|g\|_{H}<R\right\}$ and let $h \in B_{R}$. Take an arbitrary $\epsilon>0$. There exists a number $N>0$ such that $\sum_{n=N+1}^{\infty}\left\|e_{n}-h_{n}\right\|_{H}^{2}<\epsilon$, therefore

$$
\begin{aligned}
\left\|F-\sum_{n=0}^{N} a_{n}\left(e_{n}-h_{n}\right)\right\|_{H}^{2}= & \left\|\sum_{n=N+1}^{\infty} a_{n}\left(e_{n}-h_{n}\right)\right\|_{H}^{2} \leqslant \\
& \leqslant \sum_{n=N+1}^{\infty} a_{n}^{2} \cdot \sum_{n=N+1}^{\infty}\left\|e_{n}-h_{n}\right\|_{H}^{2} \leqslant M^{2} R^{2} \epsilon .
\end{aligned}
$$

Hence, in view of the arbitrariness of $\epsilon>0$, the set $U\left(B_{R}\right)$ is relatively compact in $H$ and thus, the complete continuity of the operator $U$ in $H$ is proved.

Denote $\varphi=h-F$ and $B=I d-U$, where $I d$ is the identity. Then, $\varphi=\sum_{n=0}^{\infty} a_{n} h_{n}, B$ is a bounded linear operator in $H$ and $\varphi=B h$. But the equation $B h=0$ has in $H$ only the trivial solution $h=0$ because the system $\left\{h_{n}\right\}_{n=0,1,2, \ldots}$ 
is linearly independent in $H$. Therefore, the operator $B$ has a bounded inverse $B^{-1}$ in $H$; in addition, clearly $B e_{n}=h_{n}$.

Let $u \in H$ and $v=B^{-1} u=\sum_{n=0}^{\infty} a_{n} e_{n}$, where $a=\left(a_{0}, a_{1}, \ldots, a_{n}, \ldots\right) \in l_{2}$. Then, $u=B v=\sum_{n=0}^{\infty} a_{n} B e_{n}=\sum_{n=0}^{\infty} a_{n} h_{n}$ in $H$. Conversely, if $u=\sum_{n=0}^{\infty} a_{n} h_{n}$, then $B^{-1} u=\sum_{n=0}^{\infty} a_{n} e_{n}$ so that $a=\left(a_{0}, a_{1}, \ldots, a_{n}, \ldots\right) \in l_{2}$. Our proof of the Bari theorem is complete.

Remark 2.4. It immediately follows from our proof of the Bari theorem that if $\left\{h_{n}\right\}_{n=0,1,2, \ldots}$ is a Riesz basis in $H$, then there exist $0<c<C$ such that

$$
c\|a\|_{l_{2}}^{2} \leqslant\left\|\sum_{n=0}^{\infty} a_{n} h_{n}\right\|_{H}^{2} \leqslant C\|a\|_{l_{2}}^{2}
$$

for any $a=\left(a_{0}, a_{1}, \ldots, a_{n}, \ldots\right) \in l_{2}$. Indeed, the left-hand side estimate is already proved, and the right-hand side one follows the Banach theorem because as it is proved, the correspondence $h \rightarrow a$ as a map from $H$ into $l_{2}$ is linear, bounded and one-to-one, hence the inverse mapping is linear and bounded, too.

Corollary 2.5. Let again $H$ be a separable Hilbert space over the field of real numbers, $\left\{e_{n}\right\}_{n=0,1,2, \ldots}$ be an orthonormal basis in $H$ and a system $\left\{h_{n}\right\}_{n=0,1,2, \ldots} \subset H$ be such that $\sum_{n=0}^{\infty}\left\|e_{n}-h_{n}\right\|_{H}^{2}=a<1$. Then, $\left\{h_{n}\right\}_{n=0,1,2, \ldots}$ is a Bari basis in $H$.

Proof. In view of the Bari theorem 2.3 we need only to prove the linear independence of the system $\left\{h_{n}\right\}_{n=0,1,2, \ldots}$ in $H$. Suppose that $\sum_{n=0}^{\infty} c_{n} h_{n}=0$ in $H$ for some real coefficients $c_{n}$. For any integer $N>0$, we have $\sum_{n=0}^{N} c_{n}\left(e_{n}-h_{n}\right)+$ $+\alpha_{N}=\sum_{n=0}^{N} c_{n} e_{n}$ in $H$, where $\left\|\alpha_{N}\right\|_{H} \rightarrow 0$ as $N \rightarrow \infty$. Hence,

$$
\begin{aligned}
a^{1 / 2}\left(\sum_{n=0}^{N} c_{n}^{2}\right)^{1 / 2}+\left\|\alpha_{N}\right\|_{H} \geqslant\left(\sum_{n=0}^{N} c_{n}^{2}\right)^{1 / 2} & \left(\sum_{n=0}^{N}\left\|e_{n}-h_{n}\right\|_{H}^{2}\right)^{1 / 2}+\left\|\alpha_{N}\right\|_{H} \geqslant\left(\sum_{n=0}^{N} c_{n}^{2}\right)^{1 / 2}
\end{aligned}
$$

for any $N$, which is possible if and only if $0=c_{0}=c_{1}=\ldots=c_{n}=\ldots$. 
Remark 2.6. Riesz and Bari bases being compared with arbitrary bases possess a number of additions important properties. In particular, a Riesz basis after an arbitrary permutation of its elements remains the property to be a Riesz basis. For us, these additional properties of Riesz and Bari bases are less important because we only try to answer the principal question whether eigenfunctions of nonlinear problems form bases in some cases; we refer readers to the Bari article [2] and to monograph [6] for an information about these properties of Riesz and Bari bases.

2.2. Sufficient Conditions for a System of Functions to Be a Riesz Basis in $L_{2}$. A Counterexample. In this subsection, first, we shall reestablish a result on the subject indicated in the title published in [21].

Theorem 2.7. Let $\left\{h_{n}\right\}_{n=0,1,2, \ldots}$ be a sequence of real-valued, three times continuously differentiable functions in $\mathbb{R}$. Assume that for each integer $n \geqslant 0$ the following holds:

(1) $h_{n}(x+1 /(n+1))=-h_{n}(x)$ and $h_{n}(1 / 2(n+1)+x)=h_{n}(1 / 2(n+1)-x)$ for any $x \in \mathbb{R}$;

(2) $h_{n}^{\prime}(x)>0, h_{n}^{\prime \prime}(x) \leqslant 0$ and $h_{n}^{\prime \prime \prime}(x) \leqslant 0$ for any $x \in(0,1 / 2(n+1))$;

(3) there exist $0<c<C$ such that $c<h_{n}(1 / 2(n+1))<C$ for all $n$. Then, the system $\left\{h_{n}\right\}_{n=0,1,2, \ldots}$ is a Riesz basis in $L_{2}$.

Corollary 2.8. Let $\left\{h_{n}\right\}_{n=0,1,2, \ldots}$ be a sequence of real-valued, three times continuously differentiable functions in $\mathbb{R}$ satisfying assumptions (1) and (2) of the previous theorem. Then, $\left\{h_{n}\right\}_{n=0,1,2, \ldots}$ is a basis and $\left\{h_{n} / h_{n}(1 / 2(n+\right.$ 1)) $\}_{n=0,1,2, \ldots}$ is a Riesz basis in $L_{2}$.

Corollary 2.9. Let $h$ be a real-valued, three times continuously differentiable function in $\mathbb{R}$, such that $h(x+1)=-h(x), h(1 / 2+x)=h(1 / 2-x), h^{\prime}(x)>0$, $h^{\prime \prime}(x) \leqslant 0$ and $h^{\prime \prime \prime}(x) \leqslant 0$ in $(0,1 / 2)$. Then, the functions $h_{n}(x)=h((n+1) x)$, where $n=0,1,2, \ldots$, form a Riesz basis in $L_{2}$.

Proof of theorem 2.7. Denote $e_{n}(x)=\sqrt{2} \sin \pi(n+1) x, n=0,1,2, \ldots$, so that $\left\{e_{n}\right\}_{n=0,1,2, \ldots}$ is an orthonormal basis in $L_{2}$. In addition, note that $h_{n}(0)=0$ for each integer $n \geqslant 0$ by conditions (1) and (2) of the theorem.

Lemma 2.10. Let for some integer $n \geqslant 0$ a function $h_{n} \equiv g$ satisfy assumption (1) of theorem 2.9 and $g(0)=0$. Then,

$$
g(\cdot)=\sum_{m=0}^{\infty} c_{m} e_{m}(\cdot) \text { in } L_{2},
$$

where real coefficients $c_{m}$ are such that $c_{0}=c_{1}=\ldots=c_{n-1}=0$. If in addition $g(x)>0$ for $x \in(0,1 /(n+1))$, then $c_{n}>0$.

Proof. The sequence $\left\{e_{(n+1)(l+1)-1}\right\}_{l=0,1,2, \ldots}$ is an orthogonal basis in $L_{2}(0,1 /(n+1))$. Hence, the expansion above holds in the latter space with $c_{m}=0$ if $m \neq(n+1)(l+1)-1$ for all integer $l \geqslant 0$ (so that in particular $\left.c_{0}=c_{1}=\ldots=c_{n-1}=0\right)$. In addition, $c_{n}>0$ if $g(x)>0$ in $(0,1 /(n+1))$ 
because in this case two functions $e_{n}$ and $g$ are of the same sign everywhere in $(0,1 /(n+1))$. In view of the oddness of functions $g$ and $e_{(n+1)(l+1)-1}$ with respect to their zeros $\frac{k}{n+1}, k=0, \pm 1, \pm 2, \ldots$, the expansion still holds in $L_{2}$.

So, for the system $\left\{h_{n}\right\}_{n=0,1,2, \ldots}$, we have the following sequence of expansions in $L_{2}$ :

$$
h_{n}=a_{m}^{n} e_{m},
$$

where

$$
\begin{aligned}
a_{0}^{n}=a_{1}^{n}=\ldots= & a_{n-1}^{n}=0, a_{m}^{n}=0 \text { if } m \neq \\
& \neq(n+1)(l+1)-1 \text { for all } l=0,1,2, \ldots \text { and } a_{n}^{n}>0 .
\end{aligned}
$$

Denote $A=\left(a_{m}^{n}\right)_{n, m=0,1,2, \ldots}$.

Lemma 2.11. Under the assumptions of theorem 2.7, one has: $\left(a_{n}^{n}\right)^{-1}\left|a_{(n+1)(m+1)-1}^{n}\right| \leqslant \frac{\pi}{2}(m+1)^{-2}$ from each integer $m \geqslant 1$. In addition, $a_{(n+1)(m+1)-1}^{n}=0$ if $m=2 l+1$ for some integer $l \geqslant 0$.

Proof. The second claim is obvious because the functions $e_{(n+1)(2 l+2)-1}$ are odd with respect to the middles of the intervals $(0,1 /(n+1)),(1 /(n+1)$, $2 /(n+1)), \ldots,(n /(n+1), 1)$ and $h_{n}$ is even so that $a_{(n+1)(m+1)-1}^{n}=$ $=\left(e_{(n+1)(2 l+2)-1}, h_{n}\right)=0$.

Let us prove the first claim. Let $m=2 l$. Due to the properties of periodicity, oddness and evenness of $h_{n}$ and $e_{(n+1)(2 l+1)-1}$, we have

$$
\begin{aligned}
& \left(a_{n}^{n}\right)^{-1}\left|a_{(n+1)(m+1)-1}^{n}\right|=\frac{\left|\int_{0}^{1} h_{n}(x) \sin \pi(n+1)(m+1) x d x\right|}{\int_{0}^{1} h_{n}(x) \sin \pi(n+1) x d x}= \\
& =\frac{\left|\int_{0}^{1 / 2(n+1)} h_{n}(x) \sin \pi(n+1)(m+1) x d x\right|}{\int_{0}^{1 / 2(n+1)} h_{n}(x) \sin \pi(n+1) x d x}= \\
& =(m+1)^{-1} \frac{\left|\int_{0}^{1 / 2(n+1)} h_{n}^{\prime}(x) \cos \pi(n+1)(m+1) x d x\right|}{\int_{0}^{1 / 2(n+1)} h_{n}^{\prime}(x) \cos \pi(n+1) x d x}=
\end{aligned}
$$

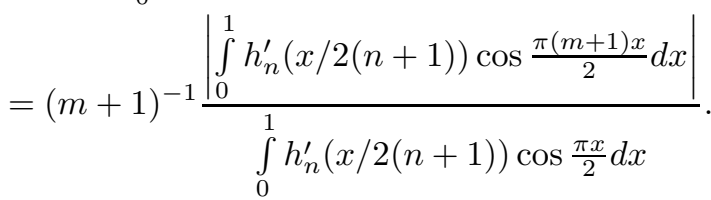


By our assumptions, $h_{n}^{\prime}(x / 2(n+1))$ is a positive nonincreasing concave function in $(0,1)$. Hence,

$$
\int_{0}^{1} h_{n}^{\prime}(x / 2(n+1)) \cos \frac{\pi x}{2} d x \geqslant h_{n}^{\prime}(0) \int_{0}^{1}(1-x) \cos \frac{\pi x}{2} d x=\frac{4}{\pi^{2}} h_{n}^{\prime}(0) .
$$

By analogy, as one can easily see on the graph of $h_{n}^{\prime}$,

$$
\begin{aligned}
& \left|\int_{0}^{1} h_{n}^{\prime}(x / 2(n+1)) \cos \frac{\pi(m+1) x}{2} d x\right| \leqslant \\
& \leqslant h_{n}^{\prime}(0)\left|\int_{0}^{1 /(m+1)} \cos \frac{\pi(m+1) x}{2} d x\right|=\frac{2}{\pi(m+1)} h_{n}^{\prime}(0) .
\end{aligned}
$$

(Indeed, denoting $x_{k}=\frac{2 k+1}{m+1}, k=0,1, \ldots, \frac{m}{2}$, and representing the latter integral as a sum of the integrals between 0 and $x_{0}, x_{0}$ and $x_{1}$, etc., one sees that the absolute value of the first integral in this sum is not smaller than the one half of the absolute value of the second one, the absolute value of the second integral is not smaller than the absolute value of the third one, and so on, which yields our estimate.)

Estimates (2.3)-(2.5) imply

$$
\left(a_{n}^{n}\right)^{-1}\left|a_{(n+1)(m+1)-1}^{n}\right| \leqslant \frac{\pi}{2}(m+1)^{-2},
$$

and lemma 2.11 is proved.

Lemma 2.12. Under the assumptions of our theorem the system $\left\{h_{n}\right\}_{n=0,1,2, \ldots}$ is linearly independent in $L_{2}$.

Proof. On the contrary, suppose that $\sum_{n=0}^{\infty} d_{n} h_{n}=0$ in $H$ for some $d_{n} \in \mathbb{R}$. Take the scalar product of the left- and right-hand sides of this equality with $e_{0}$ in $L_{2}$. Then, it follows from (2.1) that $d_{0}=0$. By analogy, multiplying this equality by $e_{1}, e_{2}, \ldots$ in $L_{2}$ we deduce, step by step, that $0=d_{1}=d_{2}=\ldots=d_{n}=\ldots$.

For each integer $m \geqslant 0$, denote by $B_{m}$ the linear operator in $L_{2}$ mapping $e_{n}$ into $b_{(n+1)(m+1)-1}^{n} e_{(n+1)(m+1)-1}, \quad n=0,1,2, \ldots$, where $b_{m}^{n}=\left(a_{n}^{n}\right)^{-1} a_{m}^{n}$. Then, for each $m, B_{m}$ is a bounded linear operator from $L_{2}$ into $L_{2},\left\|B_{m}\right\| \leqslant$ $\sup _{n \geqslant 0}\left|b_{(n+1)(m+1)-1}^{n}\right|=b_{m}$ and $B_{0}=I d$. By lemma 2.11,

$$
\sum_{m=1}^{\infty} b_{m} \leqslant \frac{\pi}{2} \sum_{l=1}^{\infty}(2 l+1)^{-2} \leqslant \frac{\pi}{2} \int_{1 / 2}^{\infty}(2 x+1)^{-2} d x=\frac{\pi}{8} .
$$


Hence, $\|B\| \leqslant \frac{\pi}{8}<1$, where $B=\sum_{m=1}^{\infty} B_{m}$. Therefore, the operator $A=(I d+B)$ has a bounded inverse $A^{-1}=I d+\sum_{n=1}^{\infty}(-1)^{n} B^{n}$. Note also that $A e_{n}=\bar{h}_{n}$, where $\bar{h}_{n}=\left(a_{n}^{n}\right)^{-1} h_{n}$.

Take an arbitrary $v \in L_{2}$ and set $u=A^{-1} v=\sum_{n=0}^{\infty} c_{n} e_{n}$, where $c_{n} \in \mathbb{R}$. Then, $\sum_{n} c_{n}^{2}<\infty$ and $v=A u=\sum_{n=0}^{\infty} c_{n} A e_{n}=\sum_{n=0}^{\infty} c_{n} \bar{h}_{n}$, where the infinite sums converge in $L_{2}$. Therefore, in view of lemma 2.12 , the system $\left\{h_{n}\right\}_{n=0,1,2, \ldots}$ is a basis in $L_{2}$, and, if $\sum_{n} c_{n}^{2}<\infty$, then the series $\sum_{n=0}^{\infty} c_{n} \bar{h}_{n}$ converges in $L_{2}$.

Conversely, let a series $u=\sum_{n=0}^{\infty} c_{n} \bar{h}_{n}$ converges in $L_{2}$. Then, $A^{-1} u=$ $\sum_{n} c_{n} e_{n}$, hence $\sum_{n} c_{n}^{2}<\infty$. Thus, $\left\{\bar{h}_{n}\right\}_{n=0,1,2, \ldots}$ is a Riesz basis in $L_{2}$. Since under the assumptions of the theorem we easily have $0<c \leqslant a_{n}^{n} \leqslant C<+\infty$ for some constants $0<c<C$ independent of $n$, our proof of theorem 2.7 is complete.

Remark 2.13. In the following, theorem 2.7 will be applied to prove theorem 1.3. So, one can see that the set of all Riesz (or Bari) bases in $L_{2}$ is essentially wider than the the set of systems of solutions of our nonlinear problems. However, the same remark still concerns linear problems: the set of all bases in $L_{2}$ is essentially wider than the set of sequences of eigenfunctions of linear problems.

In our proof of theorem 2.7, we essentially used the fact that the matrix $A$ in the expansions (2.1)-(2.2) is upper triangular and all elements of its principal diagonal are strictly positive. So, one may think that in view of these properties of the matrix $A$, the sequence $\left\{h_{n}\right\}_{n=0,1,2, \ldots}$ is always a basis in $L_{2}$. The following counterexample taken from our paper [24] shows that this is not so and, generally speaking, the system $\left\{h_{n}\right\}_{n=0,1,2, \ldots}$ can be even incomplete.

Example 2.14. Let $\left\{e_{n}\right\}_{n=0,1,2, \ldots}$ be an orthonormal basis in the space $H$ introduced earlier, and the system $\left\{h_{n}\right\}_{n=0,1,2, \ldots} \subset H$ is defined by formulas (2.1)-(2.2) where, for each $n, a_{n}^{n}=a, a_{n+1}^{n}=-\sqrt{1-a^{2}}, a \in(0,1)$ does not depend on $n$, and $a_{m}^{n}=0$ for all other values of $n$ and $m$. Fix $a$ so small that $A=\sqrt{1-a^{2}} / a>3$ and let us show that the system $\left\{h_{n}\right\}_{n=0,1,2, \ldots}$ is incomplete in $H$ (note that according to lemma 2.12 this system is linearly independent in 
$H)$. For this aim, let us prove that there exists a constant $c>0$ so that

$$
\left\|c_{0} h_{0}+c_{1} h_{1}+\ldots+c_{N} h_{N}-e_{0}\right\|_{H} \geqslant c
$$

for any real coefficients $c_{n}$ and any number $N$. On the contrary, suppose that this is not so and there exist sequences of numbers $N_{m}$ and of real coefficients $c_{0}^{m}, c_{1}^{m}, \ldots, c_{N_{m}}^{m}$ so that $\left\|\alpha_{m}\right\|_{H} \rightarrow 0$ as $m \rightarrow \infty$, where $\alpha_{m}=c_{0}^{m} h_{0}+c_{1}^{m} h_{1}+$ $\ldots+c_{N_{m}}^{m} h_{N_{m}}-e_{0}$.

Denote $\gamma_{m, n}=\left(\alpha_{m}, e_{n}\right)_{H}$. Then, it is easy to derive that

$$
c_{0}^{m}=a^{-1}\left(1+\gamma_{m, 0}\right) \quad \text { and } \quad c_{n}^{m}=A c_{n-1}^{m}+a^{-1} \gamma_{m, n}, n \geqslant 1 .
$$

Since $\alpha_{m} \rightarrow 0$ in $H$, we have

$$
\sum_{n} \gamma_{m, n}^{2} \rightarrow 0 \text { as } m \rightarrow \infty
$$

Hence, $c_{0}^{m} \rightarrow a^{-1}>1$ as $m \rightarrow \infty$, and applying induction in $n$ to (2.6) and the facts that $A>3$ and, by (2.7), that $\left|\gamma_{m, n}\right| \leqslant a$ for all $n$ and all sufficiently large $m$, we obtain: $c_{n}^{m}>1$ for all sufficiently large $m$ and for all $n=0,1, \ldots, N_{m}$. But then, $\left\|\alpha_{m}\right\|_{H}^{2} \geqslant\left(c_{N_{m}}^{m}\right)^{2}\left(1-a^{2}\right)>1-a^{2}>0$ for all sufficiently large $m$, and we arrive at a contradiction, which completes our proof.

In the next sections, we need in the following technical result.

Proposition 2.15. Consider the problem

$$
w^{\prime \prime}=g(w), \quad w=w(x), x \in \mathbb{R},
$$

where $g$ is a continuously differentiable function of its argument. Let $w(x)$ be a solution of (2.8). Then

(a) if $w^{\prime}\left(x_{0}\right)=0$ for some $x_{0} \in \mathbb{R}$, then the solution $w$ is even with respect to $x_{0}$, that is, $w\left(x_{0}+x\right)=w\left(x_{0}-x\right)$ for any $x$;

(b) if in addition the function $g$ is odd and if $w\left(x_{0}\right)=0$ at some $x_{0} \in \mathbb{R}$, then this solution $w$ is odd with respect to this point, that is, $w\left(x_{0}+x\right)=-w\left(x_{0}-x\right)$ for any $x$.

Proof is elementary. Let us prove, for example, claim (a) (claim (b) can be proved by the complete analogy). Let $w^{\prime}\left(x_{0}\right)=0$ and introduce the function $w_{1}(x)$ equal to $w(x)$ for $x \leqslant x_{0}$ and equal to $w\left(x_{0}-\left(x-x_{0}\right)\right)$ if $x>x_{0}$. Then, the direct verification shows that this new function $w_{1}$ is continuous everywhere and that it satisfies the differential equation in (2.8) in the domains $x \leqslant x_{0}$ and $x \geqslant x_{0}$. Thus, by the uniqueness theorem for (2.8) with the initial data $w_{1}\left(x_{0}\right)=w\left(x_{0}\right), w_{1}^{\prime}\left(x_{0}\right)=0$, one has $w \equiv w_{1}$, and proposition 2.15 is proved. 


\section{SEMILINEAR EIGENVALUE PROBLEMS IN A FINITE INTERVAL}

In this section, we shall consider nonlinear problems containing a spectral parameter.

3.1. A Proof of Brown's Result. In this subsection, we shall analyze only problem (1.1a)-(1.2) because problem (1.1b)-(1.2) can be studied by the complete analogy. Consider the linear eigenvalue problem

$$
\begin{gathered}
-\left(p e^{\prime}\right)^{\prime}+q(x) e=\lambda e, \quad e=e(x), x \in(0,1), \\
a_{1} e(0)+a_{2} e^{\prime}(0)=0=b_{1} e(1)+b_{2} e^{\prime}(1) .
\end{gathered}
$$

It has an infinite sequence of eigenvalues $\left\{\lambda_{k}\right\}_{k=0,1,2, \ldots}$ with corresponding eigenfunctions $e_{k}, k=0,1,2, \ldots$, normalized to 1 in $L_{2}$ so that $\left\{e_{k}\right\}_{n=0,1,2, \ldots}$ is an orthonormal basis in $L_{2}$. In addition, to each integer $k \geqslant 0$ the eigenfunction $e_{k}$ possesses precisely $k$ zeros in $(0,1)$.

Lemma 3.1. Take an arbitrary integer $k \geqslant 0$. There exists an interval $I_{k} \ni 0$ such that for each $\alpha \in I_{k}, \alpha \neq 0$, there exists an eigenfunction $u_{k}$ of problem (1.1a), (1.2) which possesses precisely $k$ zeros in the interval $(0,1)$ and satisfies the estimate $\left|u_{k}-\alpha e_{k}\right|_{2}=\alpha \gamma_{k}(\alpha)$, where $\gamma_{k}(\alpha) \rightarrow 0$ as $\alpha \rightarrow 0$. The corresponding eigenvalue goes to $\lambda_{k}$ as $\alpha$ goes to 0.

Remark 3.2. In fact, in [3] the author applies a stronger result, the CrandallRabinowitz bifurcation theorem, taken from [5]. However, these two approaches, our one and the approach in [3], in the context of our problem lead us to the same goal. Below we shall use the fact that we deal only with ordinary second-order differential equations, and our methods are quite elementary.

Proof of lemma 3.1. Consider the Cauchy problem

$$
\begin{gathered}
-\left(p w^{\prime}\right)^{\prime}+q(x) w=\lambda\left(w+\sum_{i=1}^{n} c_{i}(x) w^{k_{i}}\right) \quad x \in(0,1), \\
a_{1} w(0)+a_{2} w^{\prime}(0)=0, a_{2} w(0)-a_{1} w^{\prime}(0)=A .
\end{gathered}
$$

By (3.4), the values $w(0)$ and $w^{\prime}(0)$ are uniquely determined and one has: $c|A| \leqslant$ $|w(0)|+\left|w^{\prime}(0)\right| \leqslant C|A|$ for all $A:|A|<1$, where positive constants $0<$ $c<C$ do not depend on $A$. Hence, by the continuous dependence theorem, $\max |w(x)| \rightarrow 0$ as $A \rightarrow 0$ for any fixed $\lambda$. Furthermore, since the absolute $x \in[0,1]$

values of the nonlinear terms in (3.3) can be estimated from above by $C_{1}|w|^{1+\kappa}$ for constants $C_{1}>0$ and $\kappa>0$ independent of sufficiently small $A \neq 0$, we obtain from (3.3) applying Gronwell's lemma:

$$
\max _{x \in[0,1]}|w(x)| \leqslant C_{2}|A|
$$

for all sufficiently small $A \neq 0$ and a constant $C_{2}>0$ independent of $A$. 
By the oscillation theorem (see, for example, [4]), for each integer $n \geqslant 0$ there exist two strictly positive functions $\alpha=\alpha(A)$ and $\beta=\beta(A)$ of the argument $A \neq 0$ going to 0 as $A \rightarrow 0$, such that for any sufficiently small $A \neq 0$ each of two solutions $w_{\alpha}$ and $w_{\beta}$ of problem (3.3)-(3.4) taken respectively with $\lambda=\lambda_{k}+\alpha(A)$ and with $\lambda=\lambda_{k}-\beta(A)$ has precisely $k$ zeros in $(0,1)$ and $\operatorname{sign}\left(b_{1} w_{\alpha}(1)+b_{2} w_{\alpha}^{\prime}(1)\right) \cdot\left(\operatorname{sign}\left(b_{1} w_{\beta}(1)+b_{2} w_{\beta}^{\prime}(1)\right)<0\right.$.

For each sufficiently small $A \neq 0$, take for $\bar{\lambda}_{k}(A)$ the largest lower bound of those values of $\lambda \in\left[\lambda_{k}-\beta(A), \lambda_{k}+\alpha(A)\right]$ for each of which the corresponding solution $w(x)$ of problem (3.3)-(3.4) has precisely $k$ zeros in $(0,1)$ and $\operatorname{sign}\left(b_{1} w(1)+b_{2} w^{\prime}(1)\right)=\operatorname{sign}\left(b_{1} w_{\alpha}(1)+b_{2} w_{\alpha}^{\prime}(1)\right)$. Then, this is a simple corollary of the oscillation and continuous dependence theorems that the solution $\bar{w}(x, A)$ of problem (3.3)-(3.4) taken with $\lambda=\bar{\lambda}_{k}(A)$ has precisely $k$ zeros in $(0,1)$ and $b_{1} \bar{w}(1, A)+b_{2} \bar{w}_{x}^{\prime}(1, A)=0$. So, we have proved that there exists a function $m=m(A)$ of sufficiently small values of $A$ going to 0 as $A \rightarrow 0$ and such that the solution $\bar{w}_{k}$ of problem (3.3)-(3.4) taken with $\lambda=\lambda_{k}+m(A)$ satisfies problem (1.1a), (1.2).

Denote by $\bar{c}_{k}=\bar{c}_{k}(A) \in \mathbb{R} \backslash\{0\}$ a constant such that $\bar{e}_{k}=\bar{c}_{k} e_{k}$ satisfies $a_{2} \bar{e}_{k}(0)-a_{1} \bar{e}_{k}^{\prime}(0)=A$. Then, by the arguments above and Gronwell's lemma, there exists $C_{3}>0$ such that for any sufficiently small $A \neq 0$ one has

$$
\max _{x \in[0,1]}\left|\bar{w}_{k}(x)-\bar{e}_{k}(x)\right| \leqslant C_{3} A^{1+\kappa} .
$$

Lemma 3.1 is proved.

Proof of the Brown theorem 1.1. We apply lemma 3.1. For each integer $k \geqslant 0$ take $\alpha=\alpha_{k} \in I_{k}, \alpha_{k} \neq 0$, so small that $\gamma_{k}^{2}\left(\alpha_{k}\right)<2^{-\frac{k+4}{2}}$. Consequently $\left|\alpha_{k}^{-1} \bar{w}_{k}(x)-e_{k}(x)\right|_{2}^{2}=\gamma_{k}^{2}\left(\alpha_{k}\right)<2^{-\frac{k+4}{2}}$. Hence,

$$
\sum_{k=0}^{\infty}\left|\alpha_{k}^{-1} \bar{w}_{k}-e_{k}\right|_{2}^{2} \leqslant \frac{1}{2}
$$

and thus, by corollary $2.5,\left\{\alpha_{k} \bar{w}_{k}\right\}_{k=0,1,2, \ldots}$ is a Bari basis in $L_{2}$. Theorem 1.1 is proved.

Remark 3.3. In fact, with theorem 1.1 we have proved the existence of a Bari basis consisting of normalized eigenfunctions of problem (1.1a), (1.2). In addition, we mention here the paper by Makin and Thompson [11] in which the authors present a stronger result on the property of being a basis for eigenfunctions of a similar problem. In their theorem, the eigenfunctions $u_{n}$ do not go to 0 uniformly in $x$ as $n$ goes to $\infty$, but these eigenfunctions are sufficiently small in a sense uniformly in $n$.

3.2. On the Existence and Uniqueness of Eigenfunctions. In this section, we shall prove a result on the existence and uniqueness of eigenfunctions of a 
nonlinear problem we need in the following. Consider the following problem:

$$
\begin{gathered}
-\frac{d^{2} u}{d x^{2}}+f(u)=\lambda u, \quad u=u(x), x \in(0,1), \\
u(0)=p>0 \\
u^{\prime}(0)=u(1)=0,
\end{gathered}
$$

where $\lambda \in \mathbb{R}$ is a spectral parameter and $p>0$ is an arbitrary fixed parameter. Our result is as follows.

Theorem 3.4. Let assumption $(f)$ be valid. Then, for any integer $n \geqslant 0$ problem (3.7)-(3.9) has a unique eigenfunction $u_{n}=u_{n}(x)$ that possesses precisely $n$ zeros in the interval $(0,1)$.

Proof. Of course, equation (3.7) can be solved by quadratures. However, below we establish its qualitative analysis which is more simple visually and applicable to wider classes of problems. In addition to problem (3.7)-(3.9), consider the Cauchy problem

$$
\begin{gathered}
-\frac{d^{2} u}{d x^{2}}+f(u)=\lambda u, \quad u=u(x), x \in(0,1), \\
u(0)=p, u^{\prime}(0)=0 .
\end{gathered}
$$

It is easy to see that if $u(x)$ is a solution of problem (3.10)-(3.11), then it satisfies the identity

$$
\frac{1}{2}\left[u^{\prime}(x)\right]^{2}+\frac{\lambda}{2}\left[u^{2}(x)-p^{2}\right]+F(p)-F(u(x))=0,
$$

where $F(u)=\int_{0}^{u} f(s) d s$. To obtain this relation, it suffices to multiply equation (3.10) by $u^{\prime}(x)$ and integrate the result from 0 to $x$. In view of this identity, it is easily seen that if a solution $u(x)$ of problem (3.10)-(3.11) is bounded in a bounded interval $[0, a)$, then it can be continued onto a right half-neighborhood of the point $x=a$.

Lemma 3.5. For any $\lambda \in \mathbb{R}$, denote by $[0, a)$ the maximal interval on which the corresponding solution of problem (3.10)-(3.11) can be continued. Then, if $u^{\prime \prime}(0)>0$, the solution $u$ satisfies $u(x) \geqslant p$ for all $x \in[0, a)$, and if $u^{\prime \prime}(0) \leqslant 0$, then $|u(x)| \leqslant p$ for any $x \in[0, a)$.

Proof. Let $u^{\prime \prime}(0)>0$. Then, $u(x)>p$ in a right half-neighborhood of the point $x=0$. Suppose that $u(\bar{x})=p$ for some $\bar{x} \in[0, a)$. By (3.12) and (3.10), it follows that $u^{\prime}(\bar{x})=0$ and $u^{\prime \prime}(\bar{x})>0$. But then, $u(x)>p$ for all $x \neq \bar{x}$ sufficiently close to $\bar{x}$. 
Now, suppose that $u^{\prime \prime}(0) \leqslant 0$. If $u^{\prime \prime}(0)=0$, then $u(x) \equiv p$ for all $x$ by the uniqueness theorem. Let $u^{\prime \prime}(0)<0$. Then, $u(x)<p$ in a right half-neighborhood of the point $x=0$. Suppose that $u(\bar{x})=p$ for some $\bar{x} \in(0, a)$. Then, as in the case $u^{\prime \prime}(0)>0$, it can be proved that $u(x)<p$ for all $x \neq \bar{x}$ sufficiently close to $\bar{x}$. This completes our proof.

Take an arbitrary integer $n \geqslant 0$ and denote by $\Lambda_{n}$ the set of all values of $\lambda \in \mathbb{R}$ such that for each of them the corresponding solution $u(x)$ of problem (3.10)(3.11) has at least $(n+1)$ zeros in the interval $(0, b)$, where $b=\min \{1 ; a\}$. By the arguments above, each of these solutions is global, that is each of them can be continued on the entire real line $x \in \mathbb{R}$. By the oscillation theorem, for any sufficiently large $\lambda>0$ the corresponding solution $u(x)$ has no less than $(n+1)$ zeros in $(0,1)$ so that $\Lambda_{n} \neq \emptyset$. In addition, by lemma 3.5 , the set $\Lambda_{n}$ is bounded from below.

Denote $\lambda_{n}=\inf \Lambda_{n}$ and consider the corresponding solution $u_{n}(x)$ of problem (3.10)-(3.11) taken with $\lambda=\lambda_{n}$. Clearly, $\left|u_{n}(x)\right| \leqslant p$ for all $x$ so that $u_{n}$ is a global solution. Let us prove that $u_{n}$ has no less than $n$ zeros in $(0,1)$ and that $u_{n}(1)=0$ (so that $u_{n}$ is an eigenfunction of problem (3.7)-(3.9)). Take a sequence $\left\{\lambda^{k}\right\} \subset \Lambda_{n}, \quad k=1,2,3, \ldots$, such that $\lambda^{k} \searrow \lambda_{n}$ as $k \rightarrow \infty$. Denote by $u^{k}(x)$ the corresponding solutions of problem (3.10)-(3.11) taken with $\lambda=\lambda^{k}$. Then, each solution $u^{k}$ can be continued onto the whole interval $[0,1]$ and it has at least $(n+1)$ zeros in $(0,1)$. Observe that $u_{n}^{\prime}(x) \neq 0$ if $u_{n}(x)=0$ because otherwise $u_{n}(x) \equiv 0$ by the uniqueness theorem. Therefore, zeros of $u_{n}(x)$ are isolated and hence, $u_{n}$ has a finite number of zeros in $[0,1)$. Let $0<x_{1}<\ldots<x_{m}<1$ be these zeros. As is indicated above, $u_{n}^{\prime}\left(x_{l}\right) \neq 0$ for any $l=1,2, \ldots, m$. This immediately yields that $m \leqslant n$ because otherwise, by the continuous dependence theorem, there exists $\lambda<\lambda_{n}$ belonging to $\Lambda_{n}$ which is a contradiction. By analogy, if it would be $m<n$, then for any $\lambda$ sufficiently close to $\lambda_{n}$ the corresponding solution of problem (3.10)-(3.11) would have at most $(m+1) \leqslant n$ zeros in the interval $(0,1)$ which contradicts our definition of the set $\Lambda_{n}$. Hence, $m \geqslant n$ and thus, $m=n$. By the complete analogy, $u_{n}(1)=0$. So, we have proved, for any integer $n \geqslant 0$, the existence of an eigenfunction $u_{n}$ of problem (3.7)-(3.9) that possesses precisely $n$ zeros in the interval $[0,1)$.

Finally, let us prove, for each integer $n \geqslant 0$, the uniqueness of the eigenfunction $u_{n}$ of problem (3.7)-(3.9) that has precisely $n$ zeros in the interval $[0,1)$. On the contrary, suppose that there exists two values of $\lambda$, say $\lambda_{1}<\lambda_{2}$, such that each of the corresponding two solutions $u_{1}$ and $u_{2}$ of the Cauchy problem (3.10)-(3.11) has precisely $n$ zeros in $[0,1)$ and $u_{i}(1)=0, i=1,2$. Then, by identity (3.12), $\left|u_{1}^{\prime}\left(x_{1}\right)\right|<\left|u_{2}^{\prime}\left(x_{2}\right)\right|$ for any $x_{1}, x_{2} \in[0,1]$ such that $u_{1}\left(x_{1}\right)=u_{2}\left(x_{2}\right) \neq \pm p$. In addition, it is a simple corollary of proposition 2.15 that the zeros and the points of extremum of each solution $u_{1}$ and $u_{2}$ in $[0,1]$ are precisely the points $x_{k}=\frac{2 k+1}{2 n+1}$ and $\hat{x}_{k}=\frac{2 k}{2 n+1}$, respectively, where 
$k=0,1, \ldots, n$. But on the other hand, it follows from the arguments above that the first zero of $u_{1}$ in $(0,1)$ is strictly larger than the first zero of $u_{2}$ in the same interval. This contradiction completes our proof of theorem 3.4.

Remark 3.6. Let $u_{n}$ be the $n$th eigenfunction of problem (3.7)-(3.9) given by theorem 3.4. Then, as is proved above, the zeros and the points of extremum of $u_{n}$ in the interval $[0,1]$ are precisely the points $x_{k}^{n}=\frac{2 k+1}{2 n+1}$ and $\hat{x}_{k}^{n}=\frac{2 k}{2 n+1}$, respectively, where $k=0,1, \ldots, n$. In addition, $\left|u_{n}(x)\right| \leqslant p$ for all $x$ and $n$.

3.3. A Result on Basis Properties of Eigenfunctions. In this section, we shall consider basis properties of eigenfunctions of problem (3.7)-(3.9). Our result is the following.

Theorem 3.7. Under assumption $(f)$ the sequence of eigenfunctions $\left\{u_{n}\right\}_{n=0,1,2, \ldots}$ of problem (3.7)-(3.9) given by theorem 3.4 is a Riesz basis and the sequence $\left\{u_{n} /\left|u_{n}\right|_{2}\right\}_{n=0,1,2, \ldots}$ is a Bari basis in the space $L_{2}$.

Proof. In view of proposition 2.15 and remark 3.6 by the complete analogy with lemma 2.10, we have the following sequence of expansions:

$$
u_{n}(\cdot)=\sum_{k=0}^{\infty} a_{k}^{n} e_{k}(\cdot) \quad \text { in } L_{2}, n=0,1,2, \ldots
$$

where $e_{n}(x)=\sqrt{2} \cos \frac{\pi(2 n+1) x}{2}, n=0,1,2, \ldots$, form an orthonormal basis in $L_{2}, a_{k}^{n}$ are real coefficients, $a_{k}^{n}=0$ for any $k=0,1, \ldots, n-1$ and $a_{n}^{n}>0$. Therefore, by the complete analogy with lemma 2.12 , the system $\left\{u_{n}\right\}_{n=0,1,2, \ldots}$ is linearly independent in $L_{2}$.

Denote $\bar{e}_{n}(x)=p \cos \frac{\pi(2 n+1) x}{2}$. Clearly, $\left\{\bar{e}_{n}\right\}_{n=0,1,2, \ldots}$ is an orthogonal basis and a Riesz basis in $L_{2}$. To apply the Bari theorem, let us prove that the sequences $\left\{u_{n}\right\}_{n=0,1,2, \ldots}$ and $\left\{\bar{e}_{n}\right\}_{n=0,1,2, \ldots}$ are quadratically close to each other in $L_{2}$. The functions $\bar{e}_{n}$ are the eigenfunctions of the linear problem

$$
\begin{gathered}
-\bar{e}_{n}^{\prime \prime}=\mu_{n} \bar{e}_{n}, \quad \bar{e}_{n}=\bar{e}_{n}(x), x \in(0,1), \\
\bar{e}_{n}(0)=p, \bar{e}_{n}^{\prime}(0)=\bar{e}_{n}(1)=0,
\end{gathered}
$$

where $\mu_{n}=\left(\frac{\pi(2 n+1)}{2}\right)^{2}, n=0,1,2, \ldots$. In addition, since by remark 3.6 one has $\left|u_{n}(x)\right| \leqslant p$ for all $x$ and $n$ and by the comparison theorem

$$
\left|\lambda_{n}-\mu_{n}\right| \leqslant C_{1}, \quad n=0,1,2, \ldots,
$$

where $C_{1}=\sup _{u \in(0, p]}\left|\frac{f(u)}{u}\right|$. 
Set $w_{n}=u_{n}-\bar{e}_{n}$. By (3.13) and the estimate $\left|u_{n}(x)\right| \leqslant p$,

$$
\begin{gathered}
-w_{n}^{\prime \prime}+U_{n}(x)=\mu_{n} w_{n}, \quad w_{n}=w_{n}(x), x \in(0,1), \\
w_{n}(0)=w_{n}^{\prime}(0)=w_{n}(1)=0,
\end{gathered}
$$

where $U_{n}(x)=f\left(u_{n}(x)\right)+\left(\lambda_{n}-\mu_{n}\right) u_{n}(x)$ so that $\left|U_{n}(x)\right| \leqslant C_{2}$ for a constant $C_{2}>0$ independent of $x$ and $n$. In addition, by identity (3.12),

$$
\left|w_{n}^{\prime}(1)\right| \leqslant C_{3}
$$

for a constant $C_{3}>0$ independent of $n$ (for the functions $\bar{e}_{n}$, an identity analogous to (3.12) takes place).

Multiply equation (3.14) by $2 x w_{n}^{\prime}(x)$ and integrate the result over the interval $[0,1]$. Then, after an integration by parts, using (3.15), we obtain:

$$
\mu_{n} \int_{0}^{1} w_{n}^{2}(x) d x=-2 \int_{0}^{1} x w_{n}^{\prime}(x) U_{n}(x) d x-\int_{0}^{1}\left[w_{n}^{\prime}(x)\right]^{2} d x+\left[w_{n}^{\prime}(1)\right]^{2} .
$$

Thus, since $2\left|x w_{n}^{\prime}(x) U_{n}(x)\right| \leqslant\left[w_{n}^{\prime}(x)\right]^{2}+\left[U_{n}(x)\right]^{2}$ and by (3.16), we obtain $\left|w_{n}\right|_{2}^{2} \leqslant C_{4}(n+1)^{-2}$ so that indeed the systems $\left\{u_{n}\right\}_{n=0,1,2, \ldots}$ and $\left\{\bar{e}_{n}\right\}_{n=0,1,2, \ldots}$ are quadratically close to each other in the space $L_{2}$. Since this is still so for the systems $\left\{u_{n} /\left|u_{n}\right|_{2}\right\}_{n=0,1,2, \ldots}$ and $\left\{e_{n}\right\}_{n=0,1,2, \ldots}$ and in view of the Bari theorem, theorem 3.7 is completely proved.

Remark 3.8. The above proof of theorem 3.7 is taken from our papers $[19,20]$. In addition, in [20], a direct proof of this result, not based on the Bari theorem, is established.

Remark 3.9. As is noted in Introduction, a result similar to theorem 3.7 takes place if we supply equation (3.7) with one of the following two sets of boundary conditions:

$$
u(0)=u(1)=0, \quad u^{\prime}(0)=p>0
$$

or

$$
u^{\prime}(0)=u^{\prime}(1)=0, \quad u(0)=p>0,
$$

where in both cases $p>0$ is a fixed parameter. These claims are proved in [20]; their proofs are completely analogous to the above proof of theorem 3.7. In fact, the sequence of all eigenfunctions $\left\{u_{n}\right\}_{n=0,1,2, \ldots}$ of equation (3.7) taken with the second set of boundary conditions is a Riesz basis in $L_{2}$, while the sequence of all eigenfunctions of the same equations taken with the first set of boundary conditions is a basis (but not a Riesz basis) in this space. However, in each case the sequence $\left\{u_{n} /\left|u_{n}\right|_{2}\right\}_{n=0,1,2, \ldots}$ is a Bari basis in $L_{2}$. In the case of the boundary conditions of the first kind, the sequence of eigenfunctions is not a 
Riesz basis in $L_{2}$ because in this case the eigenfunctions approach zero uniformly in $x$ as $n \rightarrow \infty$, therefore, since the system $\left\{u_{n} /\left|u_{n}\right|_{2}\right\}_{n=0,1,2, \ldots}$ is a Bari basis in $L_{2}$, the series $\sum_{n=0}^{\infty} a_{n} u_{n}$ converges in $L_{2}$ not only when $\left(a_{0}, a_{1}, \ldots, a_{n}, \ldots\right) \in l_{2}$.

Remark 3.10. As for theorem 1.2 in Introduction, the proof of its principal claim (c) in $[17,25]$ is completely analogous to the above proof of theorem 3.7. Claims (a) and (b) of this theorem are proved, for example, in [16, 25]; these are technical results, their proofs are sufficiently cumbersome and therefore, we do not consider them here.

\section{FURTHER RESULTS}

In this section, we shall consider two results on the subject the paper is devoted to. First, we shall prove theorem 1.3. As was already noted in Introduction, one of the main difficulties arising in this case consists in the fact that a linear self-adjoint problem whose eigenfunctions would be arbitrary close to the solutions of problem (1.7) for $n$ sufficiently large is unknown. We shall overcome this difficulty by applying theorem 2.7 and, then, the Bari theorem 2.3. With this approach, we follow our article [21] in which in fact a bit more general result is obtained. Second, we shall discuss briefly a result published in [24] on the expansions over eigenfunctions of an equation of the type of (1.3) in the half-line $[0,+\infty)$. In this case the spectrum of the problem fills a half-line $[\bar{\lambda},+\infty)$ and an analog of the Fourier transform occurs: «an arbitrary function» can be uniquely expanded in an improper integral over eigenfunctions of the nonlinear problem similar to the representation of this function by its Fourier transform. In the present paper, we shall not establish a complete proof of this result, but we only shall sketch main idea of this proof. We refer the reader to our paper [24] for the complete proofs.

4.1. A Problem without a Spectral Parameter: Proof of Theorem 1.3. In this subsection, we shall prove theorem 1.3. For the convenience of readers, we repeat the statement of the problem:

$$
\begin{gathered}
-u^{\prime \prime}=f(u), \quad u=u(x), x \in(0,1), \\
u(0)=u(1)=0,
\end{gathered}
$$

where the nonlinearity $f$ satisfies the assumptions of theorem 1.3. The first part of this theorem, the existence of a sequence of solutions $\left\{u_{n}\right\}_{n=0,1,2, \ldots}$ so that each $n$th solution has precisely $n$ zeros in the interval $(0,1)$ is a standard and well-known result (see, for instance, [25]). Let us prove, for each integer $n \geqslant 0$, that a solution $u_{n}$ that possesses precisely $n$ zeros in the interval $(0,1)$ is unique up to the coefficient \pm 1 . 
Again, as in Sec. 3, the following identity takes place for solutions of equation (4.1):

$$
\frac{1}{2}\left[u^{\prime 2}(x)-u^{\prime}(0)^{2}\right]+[F(u(x))-F(u(0))]=0, \quad x \in[0,1],
$$

where $F(u)=\int_{0}^{u} f(s) d s$. Consequently, since $f(u) / u \rightarrow+\infty$ as $|u| \rightarrow \infty$, for any solution $u(x)$ of equation (4.1) the quantity $\left|u^{\prime}(x)\right|+|u(x)|$ is bounded in an arbitrary interval on which this solution is continuable. Therefore, an arbitrary solution of equation (4.1) is global, that is, it can uniquely continued onto the entire real line $x \in \mathbb{R}$. In addition, by proposition 2.15 , for each integer $n \geqslant 0$, the zeros and the points of extremum in $[0,1]$ of an arbitrary solution of problem (4.1)-(4.2) that has precisely $n$ zeros in the interval $(0,1)$ are precisely the points $x_{k}^{n}=\frac{k}{n+1}$, where $k=0,1, \ldots, n+1$ and $y_{k}^{n}=\frac{2 k+1}{2 n}$, where $k=0,1, \ldots, n-1$, respectively.

Now, suppose that for some integer $n \geqslant 0$ there exist two different solutions $u^{1}$ and $u^{2}$ of problem (4.1)-(4.2) each of which has precisely $n$ zeros in the interval $(0,1)$. Let for definiteness $u^{2^{\prime}}(0)>u^{1^{\prime}}(0)>0$. Then, it easily follows from identity (4.3) that $u^{2^{\prime}}\left(x_{2}\right)>u^{1^{\prime}}\left(x_{1}\right)$ for any $x_{1}, x_{2} \in\left(0, y_{1}^{n}\right)$ such that $u^{1}\left(x_{1}\right)=u^{2}\left(x_{2}\right)$. But then $u^{2}(x)>u^{1}(x)$ everywhere in $\left(0, x_{1}^{n}\right)$ and by the standard comparison theorem applied to equation (4.1) in this interval it follows that the first positive point of maximum of the solution $u^{2}$ must be smaller than that of the solution $u^{1}$ (because $f\left(u^{2}(x)\right) / u^{2}(x) \geqslant f\left(u^{1}(x)\right) / u^{1}(x)$ for any $x \in\left[0, y_{1}^{n}\right]$ and there exists a point $x$ in this interval at which this inequality is strict). This contradiction proves our claim. Thus, it is proved that for any integer $n \geqslant 0$ the solution of our problem that possesses precisely $n$ zeros in the interval $(0,1)$ is unique up to the coefficient \pm 1 .

Now, we turn to proving that the sequence $\left\{u_{n} /\left|u_{n}\right|_{2}\right\}_{0,1,2, \ldots}$ is a Riesz basis in the space $L_{2}$. By lemmas 2.10 and 2.12, this sequence is linearly independent in this space. So, to apply the Bari theorem, it suffices to construct a sequence of functions $\left\{h_{n}\right\}_{n=0,1,2, \ldots}$ that satisfies the conditions of theorem 2.7 and is quadratically close to our sequence $\left\{u_{n} /\left|u_{n}\right|_{2}\right\}_{n=0,1,2, \ldots}$ in the space $L_{2}$.

Observe that, since $f(u) / u$ is a nondecreasing function of $u>0$ and since it goes to $+\infty$ as $|u| \rightarrow \infty$, we have $f^{\prime}(u) \geqslant f(u) / u$ for all $u>0$, and therefore, there exists a constant $\bar{u}>0$ such that $f^{\prime}(\bar{u})>f^{\prime}(u), f(\bar{u})>f(u)$, $F(\bar{u})>F(u)$ for any $0<u<\bar{u}$ and, in addition, $f^{\prime}(\bar{u})>0$. We fix this choice of the constant $\bar{u}$.

Without the loss of generality, we accept that $u_{n}^{\prime}(0)>0$. By the comparison theorem, $u_{n}\left(y_{1}^{n}\right) \rightarrow+\infty$ as $n \rightarrow \infty$. For each $n$ sufficiently large, denote by $z_{n} \in\left(0, y_{1}^{n}\right)$ the point such that $u_{n}\left(z_{n}\right)=\bar{u}$. Then, $u_{n}\left(y_{1}^{n}\right)-\bar{u}=u_{n}^{\prime}\left(p_{n}\right)\left(y_{1}^{n}-z_{n}\right)$ 
for some $p_{n} \in\left(z_{n}, y_{1}^{n}\right)$. Hence, since by equation (4.1) one has $u_{n}^{\prime \prime}(x)<0$ in $\left[z_{n}, y_{1}^{n}\right]$, we obtain:

$$
u_{n}^{\prime}\left(z_{n}\right) \geqslant u_{n}^{\prime}\left(p_{n}\right)>\frac{3}{2}(n+1) u_{n}\left(y_{1}^{n}\right)
$$

for all sufficiently large $n$. In addition, since $\sup _{n} \max _{x \in\left[0, z_{n}\right]}\left|u_{n}^{\prime \prime}(x)\right|<+\infty$, we have

$$
0<z_{n} \leqslant \frac{\bar{u}}{(n+1) u_{n}\left(y_{1}^{n}\right)} \leqslant(n+1)^{-1}
$$

for all sufficiently large $n$.

For each sufficiently large $n$, we define the function $h_{n}(x)$ to be equal to $u_{n}(x)$ for any $x \in\left[z_{n}, y_{1}^{n}\right]$. To define this function in the interval $\left[0, z_{n}\right)$, we introduce two functions $g_{1}(x)$ and $g_{2}(x)$; we require that each of them is three times continuously differentiable in the interval $\left[0, y_{1}^{n}\right]$ and identically equal to $u_{n}(x)$ in $\left[z_{n}, y_{1}^{n}\right]$. In the interval $\left[0, z_{n}\right)$, we define these functions as follows. For sufficiently small $\delta_{1} \in\left(0, z_{n}\right)$, we set $g_{1}^{\prime \prime \prime}(x)=\delta_{1}^{-1}\left(x-z_{n}+\delta_{1}\right) u_{n}^{\prime \prime \prime}\left(z_{n}\right)$ in $\left[z_{n}-\delta_{1}, z_{n}\right)$ and $g_{1}(x)=0$ in $\left[0, z_{n}-\delta_{1}\right)$. Further, for a sufficiently small $\delta_{2} \in\left(0, z_{n}\right)$, take a continuous non-positive function $\omega(x)$ equal to $u_{n}^{\prime \prime \prime}(x)$ in $\left[z_{n}, y_{1}^{n}\right]$, to 0 in $\left[0, z_{n}-\delta_{2}\right]$ and such that $\int_{z_{n}-\delta_{2}}^{z_{n}} \omega(x) d x=u_{n}^{\prime \prime}\left(z_{n}\right)$ (we use here the fact that $u_{n}^{\prime \prime \prime}\left(z_{n}\right)<0$ which follows from equation (4.1)). In the interval $\left[0, z_{n}\right)$, we define $g_{2}(x)$ as a function three times continuously differentiable in $\left[0, y_{1}^{n}\right]$ and such that $g_{2}^{\prime \prime \prime}(x)=\omega(x)$ in $\left[0, z_{n}\right)$. By construction, the functions $g_{1}$ and $g_{2}$ exist and are unique.

Let us prove that if we take constants $\delta_{1}$ and $\delta_{2}$ sufficiently small, then $g_{1}(0)<0$ and $g_{2}(0)>0$. For this aim, first, observe that if $\delta_{1}>0$ is sufficiently small, then $g_{1}(0)$ is arbitrary close to

$$
u_{n}\left(z_{n}\right)-z_{n} u_{n}^{\prime}\left(z_{n}\right)+\frac{z_{n}^{2}}{2} u_{n}^{\prime \prime}\left(z_{n}\right)
$$

The latter expression is negative by construction if $\delta_{1}>0$ is sufficiently small because, by our choice of $\bar{u}$ and equation (4.1), it is smaller than

$$
0=u_{n}(0)=u_{n}\left(z_{n}\right)-z_{n} u_{n}^{\prime}\left(z_{n}\right)+\int_{0}^{z_{n}} d x \int_{x}^{z_{n}} u_{n}^{\prime \prime}(t) d t
$$

Further, for $\delta_{2}>0$ sufficiently small $g_{2}(0)$ is arbitrary close to $\bar{u}-z_{n} u_{n}^{\prime}\left(z_{n}\right)$. By (4.3) and our choice of $\bar{u}, u_{n}^{\prime}\left(z_{n}\right)<u_{n}^{\prime}(x)$ for any $x \in\left[0, z_{n}\right)$, therefore, we 
have for $\delta_{2}>0$ sufficiently small:

$$
\bar{u}=\int_{0}^{z_{n}} u_{n}^{\prime}(x) d x>z_{n} u_{n}^{\prime}\left(z_{n}\right)
$$

so that indeed $g_{2}(0)>0$ if $\delta_{2}>0$ is sufficiently small.

Fix now a choice $\delta_{1}>0$ and $\delta_{2}>0$ such that $g_{1}(0)<0$ and $g_{2}(0)>0$. Then, $\sigma g_{1}(0)+(1-\sigma) g_{2}(0)=0$ for some $\sigma \in(0,1)$. We set $h_{n}(x)=\sigma g_{1}(x)+$ $(1-\sigma) g_{2}(x)$ for all $x \in\left[0, y_{1}^{n}\right]$. Note that $h_{n}(x) \equiv u_{n}(x)$ in $\left[z_{n}, y_{1}^{n}\right]$. For all $x \in \mathbb{R}$, we continue the function $h_{n}(x)$ be the rules:

$$
h_{n}\left(y_{1}^{n}-x\right)=h_{n}\left(y_{1}^{n}+x\right) \quad \text { and } \quad h_{n}^{\prime}\left(x_{1}^{n}+x\right)=-h_{n}^{\prime}\left(x_{1}^{n}-x\right) .
$$

So, we have constructed a sequence of functions $\left\{h_{n}\right\}_{n \geqslant N}$, where $N>0$ is a sufficiently large integer number. For $n=0,1, \ldots, N-1$, we take for $h_{n}$ arbitrary functions satisfying the assumptions of corollary 2.8. Then, the sequence $\left\{\frac{h_{n}}{h_{n}\left(y_{1}^{n}\right)}\right\}_{n=0,1,2, \ldots}$ satisfies the conditions of theorem 2.7, hence, it is a Riesz basis in the space $L_{2}$.

Let us prove that the sequences $\left\{h_{n}\right\}_{n=0,1,2, \ldots}$ and $\left\{u_{n}\right\}_{n=0,1,2, \ldots}$ are quadratically close to each other in $L_{2}$. By construction, we have for $n \geqslant N$ :

$$
\max _{z \in\left[0, z_{n}\right]}\left|u_{n}^{\prime \prime}(x)\right|=\max _{u \in[0, \bar{u}]}|f(u)| \leqslant C_{1}<+\infty
$$

and

$$
\max _{x \in\left[0, z_{n}\right]}\left|h_{n}^{\prime \prime}(x)\right|=\left|h_{n}^{\prime \prime}\left(z_{n}\right)\right|=\left|u_{n}^{\prime \prime}\left(z_{n}\right)\right| \leqslant C_{1} .
$$

Therefore,

$$
\left|u_{n}(x)-h_{n}(x)\right| \leqslant C_{2} z_{n}^{2}
$$

for any $x \in\left[0, z_{n}\right]$, where the constant $C_{2}>0$ does not depend on $n$ and $x$.

Denote $\alpha_{n}=h_{n}\left(y_{1}^{n}\right)$. Then, $\alpha_{n} \rightarrow+\infty$ as $n \rightarrow \infty$ and, since each function $h_{n}$ is concave in $\left[0, y_{1}^{n}\right]$, there exist $0<c<C$ so that $c \alpha_{n} \leqslant\left|h_{n}\right|_{2} \leqslant C \alpha_{n}$ for all $n$. Using in addition (4.4), we obtain for all $n$ sufficiently large:

$$
\left|\alpha_{n}^{-1}\left(u_{n}-h_{n}\right)\right|_{2}^{2} \leqslant C_{3}(n+1)^{-5}
$$

for a constant $C_{3}>0$ independent of $n$ so that indeed the sequences $\left\{\alpha_{n}^{-1} u_{n}\right\}_{n=0,1,2, \ldots}$ and $\left\{\alpha_{n}^{-1} h_{n}\right\}_{n=0,1,2, \ldots}$ are quadratically close to each other in the space $L_{2}$. Thus, by the Bari theorem, the system $\left\{u_{n} / \alpha_{n}\right\}_{n=0,1,2, \ldots}$ is a Riesz basis in the space $L_{2}$. In view of the almost obvious estimate $\bar{c}\left|u_{n}\right|_{2} \leqslant \alpha_{n} \leqslant \bar{C}\left|u_{n}\right|_{2}$ with constants $0<\bar{c}<\bar{C}$ independent of $n$, our proof of theorem 1.3 is complete. 
Remark 4.1. Note that if $f^{\prime}(0)=0$, then, under the assumptions of theorem 1.3, it follows from equation (4.1) that, for each integer $n \geqslant 0$, one has $u_{n}^{\prime}(x)>0, u_{n}^{\prime \prime}(x) \leqslant 0$ and $u_{n}^{\prime \prime \prime}(x) \leqslant 0$ everywhere in $\left[0, y_{1}^{n}\right)$ so that in this case theorem 1.3 immediately follows from corollary 2.8 .

4.2. An Analog of the Fourier Transform Associated with a Nonlinear Problem. In this subsection, we shall consider a problem for equation (1.3) in an unbounded interval. In fact, we consider the problem

$$
\begin{gathered}
-u^{\prime \prime}+f(u)=\lambda u, \quad u=u(x), x \in(0, \infty), \\
u(0)=p>0, \\
u^{\prime}(0)=0, \sup _{x>0}|u(x)|<+\infty,
\end{gathered}
$$

where $p>0$ is an arbitrary fixed parameter and $\lambda \in \mathbb{R}$ is a spectral parameter. First of all, we discuss the notions of the eigenvalue and the corresponding eigenfunction for this problem. In our paper [24], it is accepted for simplicity that if a pair $(\lambda, u)$ consisting of a $\lambda \in \mathbb{R}$ and a real-valued function $u(x)$ twice continuously differentiable in $[0,+\infty)$ satisfies problem (4.5)-(4.7), then $\lambda$ is an eigenvalue and $u(x)$ the corresponding eigenfunction of this problem. However, the problem is nonlinear, and the equation is considered in an unbounded interval; in addition, in [24], it is found that the set of all its eigenvalues understood in the sense above (that we call the spectrum of the problem) fills a half-line $[\bar{\lambda},+\infty)$. Therefore, for this problem, even common in use approaches for defining the notions of the eigenvalue and eigenfunction are not created yet. Here, we want only to mention one of such approaches that seems to be reasonable. Given a pair $(\lambda, u)$ that satisfies equations (4.5) and (4.6) and the condition $u^{\prime}(0)=0$, one can consider the following linear eigenvalue problem:

$$
-w^{\prime \prime}+\frac{f(u)}{u} w=\mu w, \quad w=w(x), x \in(0, \infty), w^{\prime}(0)=0,
$$

where the function $\frac{f(u)}{u}$ is defined by continuity at the point $u=0$ and $\mu \in \mathbb{R}$ is a spectral parameter. Then, it can be said that if $\mu=\lambda$ and $w(x) \equiv u(x)$ are an eigenvalue and the corresponding eigenfunction of the latter linear problem, then they are an eigenvalue and the corresponding eigenfunction of the nonlinear problem (4.5)-(4.7), respectively. However, in the following we use definitions of these notions introduced in [24].

Let $\Lambda$ be the spectrum of problem (4.5)-(4.7). Denote by $S$ the Schwartz space of functions $g$ in $\mathbb{R}$ infinitely differentiable and such that for any integer $n, m \geqslant 0$ one has: $\sup _{x}\left|x^{m} \frac{d^{n} g}{d x^{n}}\right|<+\infty$. Let $S_{e}$ and $S_{o}$ be the subspaces of the space $S$ consisting respectively of even and odd functions from $S$. Below we 
denote by $u(\lambda, x)$ the eigenfunctions of problem (4.5)-(4.7) to indicate explicitly their dependence on $\lambda$. Our result for this problem is as follows.

Theorem 4.2. Let assumption $(f)$ be valid and let in addition $f$ be a ten-time continuously differentiable function in $\mathbb{R}$. Then

(a) $\Lambda=[f(p) / p,+\infty$ ) (we denote $\bar{\lambda}=f(p) / p$ );

(b) for each $\lambda \in(\bar{\lambda},+\infty)$ there exists a minimal positive zero $k(\lambda)$ of $u(\lambda, x)$ regarded as a function of $x \geqslant 0$. The correspondence $k:(\bar{\lambda},+\infty) \rightarrow(0,+\infty)$ is continuously differentiable, $k^{\prime}(\lambda)<0$ for any $\lambda \in(\bar{\lambda},+\infty), \lim _{\bar{\lambda}+0} k(\lambda)=+\infty$ and $\lim _{\lambda \rightarrow+\infty} k(\lambda)=0$. By $\lambda=\lambda(k)$ we denote the function defined in $(0, \infty)$ inverse to $k(\lambda)$;

(c) $|u(\lambda, x)| \leqslant p$ for all $x \geqslant 0$ and $\lambda \in \Lambda$;

(d) for any $g(\cdot) \in S_{e}$ there exists a function $\bar{g}(\cdot)$ continuous in $(0,+\infty)$ that satisfies the following two conditions:

(d1) there exist $C>0$ and $\gamma \in(0,1)$ such that

$$
|\bar{g}(k)| \leqslant C k^{\gamma-1}(1+k)^{-1-\gamma}
$$

for any $k>0$;

(d2) $g(x) \equiv \int_{0}^{\infty} \bar{g}(k) u(\lambda(k), x) d k$ for any $x \geqslant 0$, where the integral in the right-hand side is understood as an improper one;

(e) a continuous function $\bar{g}(\cdot)$ that satisfies properties $(d 1)$ and $(d 2)$ is unique.

Remark 4.3. Clearly, the expansion of $g(x)$ in claim (d2) is an analog of the representation of an «arbitrary function» by its Fourier transform. The author believes that the assumption that the nonlinearity $f$ is ten times continuously differentiable is technical and not necessary, and that it suffices to assume (f). However, when proving theorem 4.2 in [24], he met large technical difficulties that led him to the necessity of this additional assumption.

Now, we establish the main idea of the proof of theorem 4.2. In this result, claims (a)-(c) are technical, and they are partly proved with theorem 3.4. We focus our attention on the principal claim (d2). Denote $e(k, x)=p \cos \frac{\pi x}{2 k}$ and, for $g(\cdot) \in S_{e}$, by $\hat{g}(\cdot)$ the renormalized Fourier transform of this function so that

$$
g(x) \equiv \int_{0}^{\infty} \hat{g}(k) e\left(k^{-1}, x\right) d k .
$$

Then, according to theorem 3.7, we can uniquely expand $e(k, x)$, regarded as a function of $x$, into the series

$$
e(k, x)=\sum_{n=0}^{\infty} d_{n}(k) u\left(\frac{k}{2 n+1}, x\right)
$$


with real coefficients $d_{n}(k)$; this series converges at least in the space $L_{2}(0, k)$ (we can do this because, as is proved in [24] (and as it is easily seen), the $n$th eigenfunction $u_{n}(\cdot)$ of the problem similar to (3.7)-(3.9) but considered in the interval $(0, k)$ coincides with the eigenfunction $u\left(\frac{k}{2 n+1}, x\right)$ of problem (4.5)(4.7) regarded as a function of the second argument). Due to the properties of periodicity of $e(k, x)$ and $u\left(\frac{k}{2 n+1}, x\right)$ in $x$ following from proposition 2.15, the expansions (4.8) still hold in the spaces $L_{2}(a, b)$ for arbitrary $0 \leqslant a<b<$ $+\infty$, i.e., «almost» in the entire half-line $(0, \infty)$. Therefore, we have, quite formally, of course:

$$
\begin{gathered}
g(x)=\int_{0}^{\infty} k^{-2} \hat{g}\left(k^{-1}\right) \sum_{n=0}^{\infty} d_{n}(k) u\left(\frac{k}{2 n+1}, x\right) d k= \\
=\sum_{n=0}^{\infty} \int_{0}^{\infty} k^{-2} \hat{g}\left(k^{-1}\right) d_{n}(k) u\left(\frac{k}{2 n+1}, x\right) d k= \\
=\sum_{n=0}^{\infty} \int_{0}^{\infty} \frac{\hat{g}\left(k^{-1}(2 n+1)^{-1}\right)}{(2 n+1) k^{2}} d_{n}((2 n+1) k) u(k, x) d k= \\
=\int_{0}^{\infty}\left\{\sum_{n=0}^{\infty} \frac{\hat{g}\left(k^{-1}(2 n+1)^{-1}\right)}{(2 n+1) k^{2}} d_{n}((2 n+1) k)\right\} u(k, x) d k= \\
=\int_{0}^{\infty} \bar{g}(k) u(k, x) d k,
\end{gathered}
$$

where $\bar{g}(k)=\sum_{n=0}^{\infty} \frac{\hat{g}\left(k^{-1}(2 n+1)^{-1}\right)}{(2 n+1) k^{2}} d_{n}((2 n+1) k)$. Of course, all these equalities are completely formal so that it is not seen from them at once even that the function $\bar{g}(k)$ is well-defined. What we did in paper [24] is a justification of this sequence of formal relations. The arguments in this paper are sufficiently complicated and cumbersome and therefore, we do not establish them here; we refer the reader to the paper [24] for the complete proof of theorem 4.2.

Remark 4.4. A result analogous to theorem 4.2 occurs if one considers equation (4.5) with the boundary conditions

$$
u(0)=0, u^{\prime}(0)=p>0, \sup _{x>0}|u(x)|<+\infty .
$$

In this case, a unique expansion similar to that in theorem 4.2 (d2) of an «arbitrary function» $g(\cdot) \in S_{o}$ occurs. 


\section{REFERENCES}

1. Bari N. K. On Bases in Hilbert Space // Dokl. Akad. Nauk SSSR. 1946. V. 54, No. 5. P. 383-386 (in Russian).

2. Bari N. K. Biorthogonal Systems and Bases in Hilbert Space // Učenye Zapiski MGU. Matematika, 1951. V. 148, No.4. P. 69-107 (in Russian).

3. Brown K.J. A Completeness Theorem for a Nonlinear Problem // Proc. Edinbourgh Math. Soc. 1974. V.19, No. 2. P. 169-172.

4. Coddington E. A., Levinson N. Theory of Ordinary Differential Equations. // N. Y.; Toronto; London: McGraw-Hill Book Company, Inc., 1955.

5. Crandall M. G., Rabinowitz P.H. Bifurcation from Simple Eigenvalues // J. Func. Anal. 1971. V. 8. P. 321-340.

6. Gohberg I.Ts., Krein M. G. Introduction to the Theory of Linear Nonselfadjoint Operators. Providence, RI: AMS, 1969 (English translation).

7. Ljusternik L.A., Sobolev V.I. Elements of Functional Analysis. M.: Nauka, 1965 (in Russian).

8. Makhmudov A. P. On the Completeness of Eigenelements of Some Nonlinear Operator Equations // Dokl. Akad. Nauk SSSR. 1982. V.263, No. 1. P.23-27 (in Russian).

9. Makhmudov A.P. Basics of Nonlinear Spectral Analysis. Baku: Azerbaijan State Univ., 1984 (in Russian).

10. Makhmudov A.P. Nonlinear Eigenvalue Problems and Bifurcation Theory. Baku: ALM Publ., 1996 (in Russian).

11. Makin A. S., Thompson H. B. Convergence of Eigenfunction Expansions Corresponding to Nonlinear Sturm-Liouville Operators // Electron. J. Diff. Eqns. 2004. V.2004, No. 87. P. 1-10.

12. Pohozaev S.I. On an Approach to Nonlinear Equations // Dokl. Akad. Nauk SSSR. 1979. V.247, No. 6. P. 1327-1331 (in Russian).

13. Pohozaev S.I. A Method of Fibering for Solving Nonlinear Boundary-Value Problems // Trudy Steklov Math. Inst. 1990. V. 192. P. 146-163 (in Russian).

14. Rabinowitz P. H. Variational Methods for Nonlinear Elliptic Eigenvalue Problems // Indiana Univ. Math. J. 1974. V.23, No. 8. P.729-754.

15. Rabinowitz P.H. Minimax Methods in Critical Point Theory and Applications to Differential Equations // CBMS Regional Conf. Ser. Math. No. 65. Providence: AMS, 1986. 
16. Zhidkov P.E. Completeness of Systems of Eigenfunctions for the Sturm-Liouville Operator with Potential Depending on the Spectral Parameter and for One Nonlinear Problem // Sbornik: Mathematics. 1997. V. 188, No. 7. P. 1071-1084.

17. Zhidkov P.E. Eigenfunction Expansions Associated with a Nonlinear Schrödinger Equation. JINR Commun. E5-98-61. Dubna, 1998.

18. Zhidkov P.E. Eigenfunction Expansions Associated with a Nonlinear Schrödinger Equation on a Half-Line. JINR Preprint E5-99-144. Dubna, 1999.

19. Zhidkov P. E. Riesz Basis Property of the System of Eigenfunctions for a Nonlinear Problem of Sturm-Liouville Type // Sbornik: Mathematics. 2000. V. 191, No.3. P. $359-368$

20. Zhidkov P.E. Basis Properties of Eigenfunctions of Nonlinear Sturm-Liouville Problems // Electron. J. Diff. Eqns. 2000. V.2000, No.28. P. 1-13.

21. Zhidkov P.E. Sufficient Conditions for Functions to Form Riesz Bases in $L_{2}$ and Applications to Nonlinear Boundary-Value Problems // Electron. J. Diff. Eqns. 2001. V. 2001, No. 74. P. 1-10.

22. Zhidkov P. E. On the Property of Being a Basis for a Denumerable Set of Solutions of a Nonlinear Schrödinger-Type Boundary-Value Problem // Nonlinear Anal.: Theory, Meth. \& Appl. 2001. V.43, No.4. P.471-483.

23. Zhidkov P.E. On the Property of Being a Bari Basis for System of Eigenfunctions of a Nonlinear Integrodifferential Equation // Differentsial. Uravneniya. 2002. V.38, No. 9. P. 1183-1189 (in Russian).

24. Zhidkov P. E. An Analog of the Fourier Transform Associated with a Nonlinear OneDimensional Schrödinger Equation // Nonlinear Anal.: Theory, Methods \& Appl. 2003. V. 52, No. 3. P. 737-754.

25. Zhidkov P.E. Korteweg-de Vries and Nonlinear Schrödinger Equations: Qualitative Theory. Lecture Notes in Math. V. 1756. Heidelberg: Springer-Verlag, 2001.

Received on March 31, 2008. 


\section{Корректор T. Е. Попеко}

Подписано в печать 21.04.2008.

Формат $60 \times 90 / 16$. Бумага офсетная. Печать офсетная.

Усл. печ. л. 2,18. Уч.-изд. л. 3,08. Тираж 315 экз. Заказ № 56149.

Издательский отдел Объединенного института ядерных исследований 141980, г. Дубна, Московская обл., ул. Жолио-Кюри, 6.

E-mail: publish@jinr.ru www.jinr.ru/publish/ 